\title{
Abnormal approach-related motivation but spared reinforcement learning in MDD: Evidence from fronto-midline Theta oscillations and frontal Alpha asymmetry
}

\author{
Davide Gheza $^{1}$ (D) $\cdot$ Jasmina Bakic $^{1} \cdot$ Chris Baeken $^{2,3,4} \cdot$ Rudi De Raedt $^{5} \cdot$ Gilles Pourtois $^{1}$
}

Published online: 23 January 2019

(C) The Psychonomic Society, Inc. 2019

\begin{abstract}
Major depression is characterized by abnormal reward processing and reinforcement learning (RL). This impairment might stem from deficient motivation processes, in addition to reduced reward sensitivity. In this study, we recorded 64-channel EEG in a large cohort of major depressive disorder (MDD) patients and matched healthy controls (HC) while they performed a standard RL task. Participants were asked to discover, by trial and error, several hidden stimulus-response associations having different reward probabilities, as enforced using evaluative feedback. We extracted induced fronto-midline Theta (FMT) power timelocked to the response and feedback as neurophysiological index of RL. Furthermore, we assessed approach-related motivation by measuring frontal alpha asymmetry concurrently. At the behavioral level, MDD patients and HCs showed comparable RL. At the EEG level, FMT power systematically varied as a function of reward probability, with opposing effects found at the response and feedback levels. Although this global pattern was spared in MDD, at the feedback level these patients showed however a steep FMT power decrease across trials when reward probability was low. Moreover, they showed impaired approach-related motivation during task execution, as reflected by frontal Alpha asymmetry. These results suggest a dissociation between (globally spared) RL and (impaired) approach motivation in MDD.
\end{abstract}

Keywords Anhedonia $\cdot$ Reinforcement learning $\cdot$ Fronto-midline Theta $\cdot$ Medial frontal cortex $\cdot$ Reward prediction error

Electronic supplementary material The online version of this article (https://doi.org/10.3758/s13415-019-00693-4) contains supplementary material, which is available to authorized users.

Davide Gheza

gheza.davide@ugent.be

1 Cognitive and Affective Psychophysiology Laboratory, Department of Experimental Clinical \& Health Psychology, Ghent University, Henri Dunantlaan 2, 9000 Ghent, Belgium

2 Department of Psychiatry and Medical Psychology, Ghent University, Universitair Ziekenhuis, Ghent, Belgium

3 Department of Psychiatry, University Hospital (UZBrussel), Brussels, Belgium

4 Ghent Experimental Psychiatry (GHEP) lab, Ghent University, Ghent, Belgium

5 Psychopathology and Affective Neuroscience Laboratory, Department of Experimental Clinical \& Health Psychology, Ghent University, Ghent, Belgium

\section{Introduction}

Leading the world burden of diseases (Greden, 2001; Kessler \& Bromet, 2013), major depressive disorder (MDD) encompasses a spectrum of psychological and somatic impairments, which give rise to a large heterogeneity in terms of symptomatology, clinical course, and responsiveness to treatment. However, across all depression subtypes, a causal role in the etiology and maintenance of this disorder is usually attributed to a "diminished interest or pleasure in all, or almost all, activities" and "lack of reactivity to usually pleasurable stimuli" (DSM-V; APA, 2013), commonly referred to as anhedonia.

Several research lines have identified reward processing as a key deficit in depression, putting forward anhedonia as a valid endophenotype of this emotional disorder (Hasler, Drevets, Manji, \& Charney, 2004). Reward-related deficits in depression may correspond to alterations of multiple and nonoverlapping components (Berridge \& Robinson, 2003). These include motivation, reinforcement learning (RL), and 
hedonic capacity (Admon \& Pizzagalli, 2015), as well their interactions with specific cognitive and emotional processes. Moreover, anhedonia in depression seems to stem from an abnormal dopamine (DA)-dependent encoding of rewardrelated stimuli and RL, as well as motivation and rewardrelated decision making, more than experiencing pleasure per se (Pizzagalli, 2014; Treadway \& Zald, 2011). Consistent with this dissociation, reward does not yield the normal responsiveness to "incentive salience" and subsequent behavioral adaptation in MDD (Henriques \& Davidson, 2000). This behavioral insensitivity to reward has been linked to a poor integration of reinforcement history over time. Specifically, Pizzagalli et al., (2008; see also Vrieze et al., 2013) previously showed, using a probabilistic reward task, that MDD patients failed to develop a response bias toward more frequently rewarded stimuli or contingencies, in the absence of immediate reward delivery. Considering rewardbased decision-making, Treadway et al. (2012) elegantly showed that depressed patients were less willing to expend effort for gaining additional reward, compared with controls, highlighting a core deficit in reward anticipation and motivation in this mood disorder (see also Salamone \& Correa, 2012).

Reinforcement learning provides a standard paradigm to explore the interplay of reward processing with motivation. It corresponds to the ability to extract, by trial and error, the value of actions (Sutton \& Barto, 2018) and to approach reward-related feedback by means of specific motivational processes to eventually maximize reward. By virtue of these fundamental properties, RL allows to timely explore and characterize the nature and extent of reward-related deficits accompanying MDD (Pizzagalli, 2014). At the electrophysiological level, RL has been linked to specific DA-dependent, event-related brain potentials (ERPs), including the error[ERN] and feedback-related negativity [FRN] (Holroyd \& Coles, 2002; Yeung, Holroyd, \& Cohen, 2005). More specifically, reward prediction errors ([RPE] either response-locked for ERN or feedback-locked for FRN) are thought to be generated in deep midbrain dopaminergic structures, which in turn release or inhibit the activation of the dorsal anterior cingulate cortex (Holroyd, Pakzad-Vaezi, \& Krigolson, 2008; Proudfit, 2015; Ullsperger, Fischer, Nigbur, \& Endrass, 2014). Interestingly, the ERN is usually overactive in internalizing psychopathology (Bakic, Jepma, De Raedt, \& Pourtois, 2014; Endrass \& Ullsperger, 2014; Frank, Woroch, \& Curran, 2005; Koban \& Pourtois, 2014; Olvet \& Hajcak, 2009; Vaidyanathan, Nelson, \& Patrick, 2012; Weinberg, Riesel, \& Hajcak, 2012). Conversely the FRN, sometimes referred to as Reward Positivity (RewP), is usually blunted in MDD (Proudfit, 2015). A reduced FRN/RewP in depression could reflect a decreased reward sensitivity (Bress, Smith, Foti, Klein, \& Hajcak, 2012; Weinberg \& Shankman, 2016) as well as impaired ability to use the reinforcement history to drive implicit reward-based learning (Whitton et al., 2016).

Although the ERN and FRN/RewP have been extremely valuable to explore brain mechanisms of RL in the past (Eppinger, Kray, Mock, \& Mecklinger, 2008; Holroyd \& Coles, 2002), frontal-midline Theta oscillations (FMT, 4-8 $\mathrm{Hz}$ ) have been put forward more recently as a complementary correlate of this process (Hajihosseini \& Holroyd, 2013), bridging RPE signals with cognitive control implementation (Cavanagh, Figueroa, Cohen, \& Frank, 2012; Cavanagh \& Frank, 2014; Holroyd \& Umemoto, 2016). FMT power increases during error and negative FB processing, as well as during response conflict and unexpected events in general (Cavanagh, Frank, Klein, \& Allen, 2010; Cavanagh, Zambrano-Vazquez, \& Allen, 2012; Cohen \& Donner, 2013; Cohen, Wilmes, \& van de Vijver, 2011; Gheza, De Raedt, Baeken, \& Pourtois, 2018). During RL, it is thought to link prediction errors to behavioral adaptation and learning (Cavanagh et al., 2010; E. H. Smith et al., 2015; van de Vijver, Cohen, \& Ridderinkhof, 2014; van de Vijver, Ridderinkhof, \& Cohen, 2011), presumably by signaling the need for enhanced cognitive control (Cavanagh \& Frank, 2014) as a function of the current prediction error. In the context of RL, cognitive control includes action selection or inhibition (response level) and working memory updating according to the accumulating action-outcome history (FB level; Barch et al., 2017; Collins et al., 2017). Unlike the ERN or FRN, FMT oscillatory perturbations arising from the ACC (Cohen, Ridderinkhof, Haupt, Elger, \& Fell, 2008; Wang, 2005) reflect both phase-locked and non-phase-locked EEG activity, thereby providing a signal that is only partially captured by ERPs (e.g., the N200; Hajihosseini \& Holroyd, 2013). In accordance with this notion, Cohen and Donner (2013) previously demonstrated that removing the phaselocked component of the EEG (i.e., the ERP) did not reduce the strength of the conflict-related modulation of the residual (non-phase locked - "induced") FMT. Rather, during response conflict, the induced FMT showed stronger behavioral association with changes in response time. Moreover, compared with the ERP components, FMT may better capture neural effects associated with long-distance connections between the medial and lateral prefrontal cortex (Smith et al., 2015). By virtue of these properties, assessing induced FMT during RL may provide novel insight into reward-based learning in depression, more closely related to hedonic capacity (i.e., propensity to modulate behavior as a function of reward), and beyond DA-dependent RPE detection.

Whereas FMT oscillations provides a useful electrophysiological correlate of performance monitoring during RL, yet MDD also is characterized by core motivational deficits. More specifically, MDD is accompanied by blunted approachrelated motivation, while sometimes associated with an excessive withdrawal/avoidance behavior concurrently. 
Noteworthy, older psychophysiological research performed by Davidson and colleagues (Davidson, 1993, 1998a; Davidson, Ekman, Saron, Senulis, \& Friesen, 1990; Henriques \& Davidson, 2000) and extensively pursued over the past three decades (Coan \& Allen, 2004; Davidson, 2004; Gotlib, Ranganath, \& Rosenfeld, 1998; Eddie Harmon-Jones $\&$ Gable, 2017) showed that this approach-withdrawal motivation model explains a large amount of interindividual variability in affect styles and emotional reactivity and maps onto two competing brain systems in the frontal lobe, as expressed by hemispheric frontal asymmetries in the Alpha band, selectively. Alpha power contributing to frontal asymmetry effects is commonly reported from a set of homologous frontal leads along the coronal axis (in particular F8-F7, F6-F5, F4-F3 and F2-F1; see Stewart, Bismark, Towers, Coan, \& Allen, 2010) and is thought to be generated mostly (but not only) from the proximal dorsolateral prefrontal cortex (dIPFC) (Pizzagalli, Sherwood, Henriques, \& Davidson, 2005), even though a clear regional specificity remains difficult to establish. With regard to MDD, anhedonic symptoms, such as loss of interest, reduced hedonic capacity, and decline of goal-related motivation, have been linked to a putative hypoactive approachmotivation system, as reflected by lower left prefrontal activity at rest (Davidson, 1998b; Henriques and Davidson, 1991; Nusslock et al., 2015; Pizzagalli et al., 2005; see Thibodeau et al., 2006 for a meta-analysis) and source-estimated in the precentral and midfrontal gyri (Smith, Cavanagh, \& Allen, 2017). Although such a broad dichotomy of frontal lobes specialization might be too coarse (Miller, Crocker, Spielberg, Infantolino, \& Heller, 2013), and a recent meta-analysis showed that traditional ways of assessing Alpha asymmetry have limited diagnostic value for MDD (van der Vinne, Vollebregt, van Putten, \& Arns, 2017), recently important methodological advances have been introduced to increase the robustness and heuristic promise of this metric (Smith, Reznik, Stewart, \& Allen, 2017). Moreover, individual differences in frontal asymmetry and their association to depression seems to be more pronounced during emotionally or motivationally evocative tasks (e.g., when approach motivation is manipulated and induced; Shankman et al., 2007; Stewart et al., 2014, 2011) rather than at rest and thus may be more informative when conceived as a state response (i.e., "response capability"; Coan et al., 2006) as opposed to a trait characteristic. For instance, a recent study showed that approach motivation reflected by asymmetrical frontal cortex activation during reward anticipation distinguished depressed from never-depressed individuals and was specifically associated with motivation-related symptoms (Nelson, Kessel, Klein, \& Shankman, 2017).

In this study, we had the unique chance to assess, using behavioral and EEG methods, brain mechanisms of RL (using FMT oscillatory perturbations), as well as motivation (using frontal Alpha asymmetry) concurrently in a large cohort of treatment-resistant MDD patients and compare them to age/ sex/education-matched healthy controls. To explore RL, we capitalized on a well-validated probabilistic learning task (Eppinger et al., 2008), previously used and validated in our laboratory (Bakic et al., 2017, 2014). In short, the added value of this task is that three reward probabilities are manipulated concurrently, and their effects on the learning rate and the phasic signals of enhanced cognitive control can be explored by using appropriate EEG methods (van de Vijver et al., 2014). More specifically, learned stimulus-response associations should lead to increased FMT for incorrect responses and decreased FMT for negative FB. Based on the evidence reviewed earlier, we formulated the following hypotheses. (i) At the behavioral level, the learning slope should be steeper and accuracy higher for high compared with low reward probability, with a possible impairment of these RL-based effects in MDD patients. (ii) At the electrophysiological level, RL should be abnormal in MDD compared with controls, as evidenced by specific alterations in FMT oscillatory activity. In healthy controls, FMT should exhibit symmetric changes between response errors and negative FB as a function of reward probability (van de Vijver et al., 2014) but might be hypoactive in MDD patients, suggesting blunted cognitive control modulation during RL. However, we predicted that these group differences should likely depend on reward probability (i.e., strength of stimulus-response association), given that MDD might interfere with RL selectively when higher efforts and enhanced motivation are required to foster learning (Bakic et al., 2017; Salamone, Correa, Nunes, Randall, \& Pardo, 2012; Thomsen, 2015; Treadway et al., 2012). In particular, we expected larger group differences at the FB level when reward probability was low compared with high, because a higher motivation is presumably required in this condition for maintaining an active and sustained exploration of the FB. (iii) Core motivational processes should be impaired as well in these MDD patients. More specifically, we surmised that MDD patients, compared with the controls, would show hypo left relative to right frontal activation while processing the FB, reflecting a deficient approach-related motivation (Davidson, 1998b; Nelson et al., 2017).

\section{Material and methods}

\section{Participants}

Forty-two patients diagnosed with unipolar MDD (30 females, mean age: 41.40 , standard deviation $[\mathrm{SD}]=12.04$; meeting DSM-V criteria - American Psychiatric Association, 2013) and $60 \mathrm{HCs}$ matched on group level for age, sex, and education ( 35 females, mean age: $37.90, \mathrm{SD}=$ 12.82) participated in the current study. All participants had normal or corrected-to-normal vision. The MDD sample was 
recruited from ambulatory and hospitalized patients of the Ghent University Hospital. This EEG study was part of a larger clinical trial (http://clinicaltrials.gov/show/ NCT01832805) that examined beneficial effects of neurostimulation (accelerated intermittent theta burst stimulation [iTBS]) of the left dorsolateral prefrontal cortex (dlPFC) in MDD (see also Duprat et al., 2016). The present EEG study included baseline data collected before the start of the treatment and examined group level differences during RL between MDD patients and HCs at this specific timepoint only. The patients' diagnosis were confirmed by the Mini International Neuropsychiatric Interview (Mini; Sheehan et al., 1998). Depression severity was assessed by a certified psychiatrist with the 17-item Hamilton Rating Scale for Depression (HRSD; Hamilton, 1980) and the 21-item Beck Depression Inventory (BDI; Beck, Steer, \& Brown, 1996). Hedonic responses were assessed with self-report questionnaires, the Snaith-Hamilton Pleasure Scale (SHAPS) (Snaith et al., 1995), and the Temporal Experience of Pleasure Scale (TEPS) (Gard, Gard, Kring, \& John, 2006); the latter assessed anticipatory separately from consummatory Anhedonia. Importantly, these patients were deemed treatment-resistant (Fava, 2003) and were classified as at least Stage I treatment-resistant (i.e., they had at least one unsuccessful treatment trial with an SSRI/SNRI; Rush, Thase, \& Dubé, 2003). Moreover, all of the patients underwent a washout period from medications and were medication-free at least 2 weeks before the baseline assessment. Only habitual benzodiazepine agents were allowed. ${ }^{1}$ Exclusion criteria were (I) bipolarity, (II) the use of antipsychotics, tricyclic antidepressant, (III) a history of neurological disorders, including epilepsy and head injury with a loss of consciousness, (IV) a history of electroconvulsive therapy, (V) a past or present substance abuse, (VI) a past or present experience of psychotic episodes, and (VII) learning disorders. Some of those admitted to the study were further excluded a posteriori for the following reasons. (i) Insufficient or no learning during the main task, as indicated by learning curves below chance level ( $11 \mathrm{HCs}, 6$ MDDs). (ii) Excessively noisy EEG signal or severe EEG recording issues (3 HCs, $2 \mathrm{MDDs}$ ). (iii) Eight controls were excluded due to high or missing BDI scores. (iv) Four controls were excluded to match age and gender between HCs and MDD patients at baseline. This was achieved by removing the oldest HCs. The final sample consisted of $34 \mathrm{HCs}(27$ females, mean age: 36.21 years, $\mathrm{SD}=11.66$ ) and $34 \mathrm{MDD}$

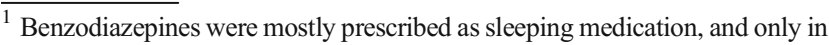
case of ongoing therapy. Possible influence of this medication on approachmotivation or RL is not documented. To note, clear frontal alpha asymmetry was previously reported in a sample of depressed patients under antidepressant medication, including lorazepam (Debener et al., 2000). Benzodiazepines administration might influence "liking" reactions, more than motivational aspects ("wanting") of the reward system (Berridge, Robinson, \& Aldridge, 2009).
}

patients ( 27 females, mean age: 42.68 years, $\mathrm{SD}=11.69$ ). The study was approved by the ethics committee of the Ghent University Hospital.

\section{Probabilistic learning task}

Participants performed a probabilistic learning task (Fig. 1) previously devised and validated by Eppinger et al. (2008) and used in Bakic et al. (2017, 2014). Colorful line drawings (Rossion \& Pourtois, 2004) were used as visual stimuli, presented against a white homogenous background on a 17 -inch computer screen. These stimuli consisted of visual objects belonging to different semantic categories (artifacts, buildings, musical instruments, clothes, vehicles, furniture). Their mean size was 7 -cm width $\mathrm{x} 5$-cm height, corresponding to $5 \mathrm{x}$ 3.6 degrees of visual angle at $80 \mathrm{~cm}$ viewing distance. On each trial, participants were required to press either the response button "A" or "B" within 800 milliseconds after stimulus onset (i.e., two-alternative forced-choice discrimination task). They were instructed to infer and learn, by trial and error, different hidden stimulus-response (S-R) mappings. Feedback on the choice made was given following every response. In each of two consecutive task blocks $(n=240$ trials each) participants were presented with six different visual stimuli, belonging to three hidden conditions that differed regarding reward probability. In each block, two stimuli had a $100 \%$ "deterministic" S-R mapping. Two stimuli had a "probabilistic" $80 \%$ S-R mapping. Finally, in the "random" S-R mapping, the two stimuli were equally often associated to each of the two response keys. Each stimulus was presented 40 times. The two different blocks differed in terms of the six visual stimuli used to avoid learning across them. Trial order within a block, as well as order of the two blocks, were alternated across participants. The trial structure was as follows: a fixation cross lasted for $250 \mathrm{~ms}$, followed by a $250-\mathrm{ms}$ blank screen. The stimulus was then presented for $500 \mathrm{~ms}$, followed by a blank screen for $300 \mathrm{~ms}$. The response time-window lasted for $800 \mathrm{~ms}$ following stimulus onset and was fixed (i.e., decisions made with response times shorter than $800 \mathrm{~ms}$ did not terminate the event). Five hundred milliseconds after response deadline, a performance feedback was presented for $500 \mathrm{~ms}$. The feedback was provided in the form of a Dutch written word, appearing in black on a white homogenous background. The word was "goed" (correct), "fout" (incorrect), or "te traag" (too late). The intertrial interval was set constant $(500 \mathrm{~ms})$ and corresponded to a blank screen. Manual responses were recorded using a Cedrus response box. Before the testing session, HCs and MDD patients were asked not to consume any caffeine or nicotine for a period of at least 2 hours. To get acquainted with the task, they completed a short practice session of 20 trials with an extra set of stimuli. The whole experiment lasted approximately 2 hours (Bakic et al., 2017). 


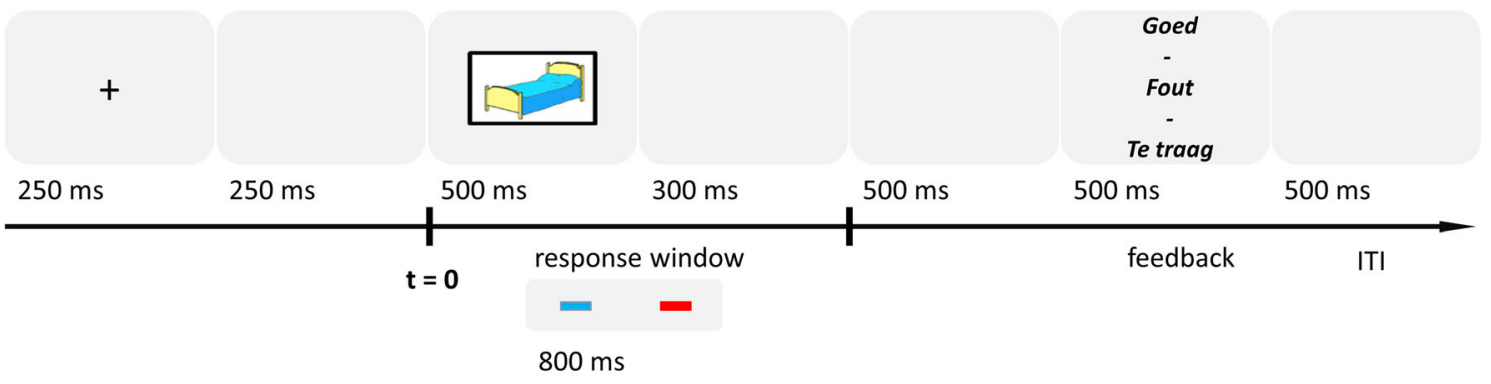

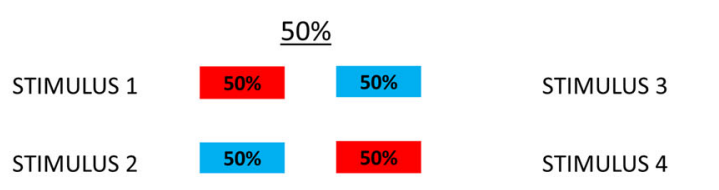

Fig. 1 (Top) Trial structure. (Bottom) The experiment consisted of two consecutive task blocks, each including 6 different stimuli that were each repeated 40 times. On each and every trial, participants were asked to perform a two-alternative forced choice task (was the stimulus associated

\section{EEG data recording, reduction and statistical analyses}

\section{EEG recording and preprocessing}

Continuous EEG was recorded during the task and sampled at $512 \mathrm{~Hz}$ using a BioSemi ActiveTwo system, with Common Mode Sense (CMS) active electrode and Driven Right Leg (DLR) passive electrode serving as ground for internal gain scaling (www.biosemi.com). A 64 channel cap, 4 periocular electrodes (above and below left eye and on left and right cantus), and 2 electrodes on the mastoids were used. The EEG signal was referenced offline to the averaged mastoids and filtered offline with a high-pass $0.5 \mathrm{~Hz}$ and low-pass $45 \mathrm{~Hz}$ FIR filters. All data processing was conducted in MATLAB (R2013; The MathWorks Inc., Natick, MA) using EEGLAB (Delorme \& Makeig, 2004) and custom scripts.

An independent component analysis was run on the continuous data. Individual epochs were then extracted around the response onset (-1.9 to $2.0 \mathrm{sec}$ ) and FB onset $(-2.4$ to $1.5 \mathrm{sec})$, and the pre-time-locking event baseline was subtracted $(-200$ to 0 ). Artefactual ICA components were selected focusing on eye artifacts and spatial or temporal discontinuities and were removed from both the FB-locked and response-locked datasets. A final dataset-wise rejection of residual epochs with artifacts was conducted by means of extreme values identification $( \pm 100 \mu \mathrm{V}$ cutoff, in a $-1,900$ to $600 \mathrm{~ms}$ time window) and visual inspection. Trials containing late responses, absence of response, or double response (both A and B button presses) were discarded from all analyses. For the probabilistic condition ( $80 \%$ feedback validity condition), trials containing unexpected feedback (i.e., $20 \%$ of trials with an inverted S-R mapping) also were removed (Bakic et al., 2014). For each dataset (response or FB), clean epochs were grouped according to the six main conditions derived by crossing the factors "reward probability" (three levels) and "accuracy"

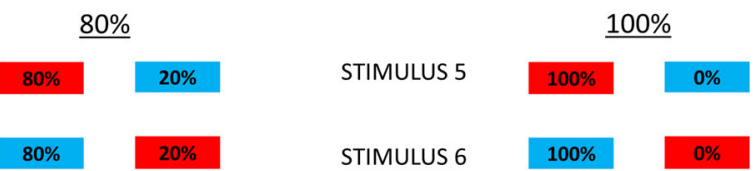

with response "A" or "B"?), within a 800-ms time limit. Unbeknown to them, these 6 stimuli were assigned to different reward probabilities (deterministic, probabilistic, or random)

(correct or incorrect response; positive or negative FB). To attenuate signal to noise ratio (SNR) differences between conditions, for each subject and dataset, conditions were balanced according to their average trial count: when a condition's count exceeded this value, a subset of epochs corresponding to this average was randomly selected. The epochs retained were included in the following analyses (individual mean and SD across conditions and datasets: $\mathrm{HCs}=52.1,16.9 ; \mathrm{MDD}=$ 48.8, 16.7. See Suppl. Table 1 for the condition-specific trial number).

\section{Time-frequency analysis}

The time-frequency decomposition was conducted using EEGLAB built-in std_ersp() function, based on complex Morlet wavelet convolution (1.6-9.85 cycles, $1.3-40 \mathrm{~Hz}, 75$ $\log$ spaced frequencies, 200 timepoints), in which the complex power spectrum of the single-trial EEG time series (obtained from FFT) was multiplied by the complex power spectrum of a family of complex Morlet wavelets, and then the inverse Fourier transform was taken (Cohen, 2014; van de Vijver et al., 2014). After convolution of the wavelets with the EEG, power was defined as the modulus of the resulting complex signal. The convolution was performed separately on feedback-locked and response-locked data. Feedback-locked and response-locked power time series were epoch-wise normalized dividing by the pre-stimulus baseline power, and decibel $(\mathrm{dB})$ converted $\left(10 * \log _{10}[\right.$ power/baseline $\left.]\right)$. The baseline interval used for the normalization was defined within the pre-stimulus interval with a fixed range for feedback-locked epochs $(-1,700$ to $-1,500 \mathrm{~ms}$ pre-FB, equal to -400 to $-200 \mathrm{~ms}$ prestimulus) and a varying range for the responselocked epochs $(-1,100$ to $-900 \mathrm{~ms}$ pre-response, equal to around -650 to $-450 \mathrm{~ms}$ prestimulus given an average response time of $\sim 450 \mathrm{~ms}$ ). The baseline for the response- 
locked epochs ensured that this range did not extend over $-100 \mathrm{~ms}$ before the stimulus presentation, even when considering the longest possible response time $(800 \mathrm{~ms})$.

Time windows and channel location were based on the theta-band maximal power from the grand average of all conditions (Fig. 2). Specifically, maximum values were reached at prefrontal scalp locations along the midline ( $\mathrm{Fz} \& \mathrm{FCz})$, in agreement with the existing RL and cognitive control literature (Cavanagh et al., 2010; Nigbur, Cohen, Ridderinkhof, \& Stürmer, 2012; van de Vijver et al., 2014). As shown in Fig. 2a, FMT power increased before the response and extended until approximately $200 \mathrm{~ms}$ after it, whereas it peaked approximately $400 \mathrm{~ms}$ after the feedback (Fig. 2b). To note, previous studies on FMT and action monitoring showed that an early FMT power burst preceding the response onset is usually expressed for both correct and incorrect responses (this comparison is shown in Supplementary Fig. 1; see also Cavanagh, Cohen, \& Allen, 2009; van de Vijver et al., 2014), whereas only incorrect responses elicit strong postresponse FMT activity (Fig. 2c). This pattern aligns well with the assumption that FMT reflects to some extent prediction error in case of response error. In line with these previous studies, FMT power was extracted in the 200-ms time window following response onset.

a

Response

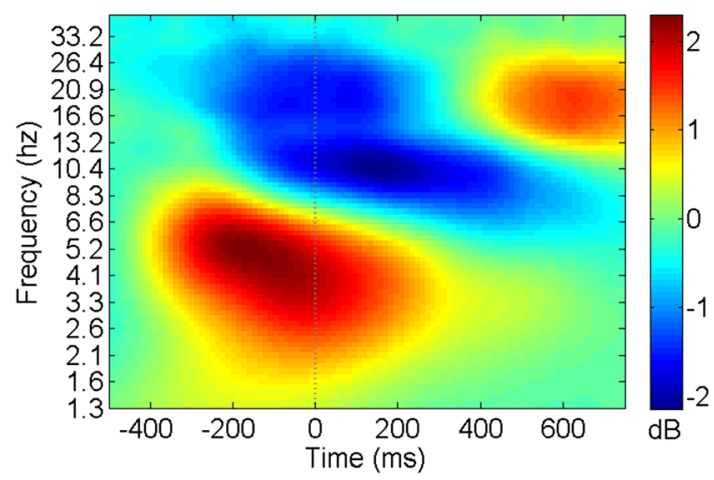

C

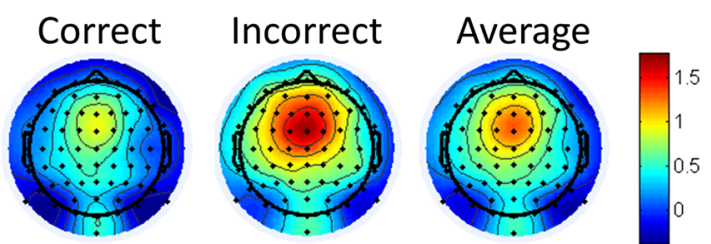

Fig. 2 Induced power. (a) Time-frequency decomposition (whole spectrum) at electrodes $\mathrm{Fz}$ and $\mathrm{FCz}$ (combined) for HCs (average of all three reward probabilities and two accuracy conditions) when considering the response level, and revealing a clear increase in FMT power $(3-7 \mathrm{~Hz})$ peaking approximately $100 \mathrm{~ms}$ before response onset and extending until approximately $200 \mathrm{~ms}$ after it. (b) Same analysis performed when considering the FB, and showing a FMT power increase occurring 300-500 $\mathrm{ms}$ after FB onset. This interval was used to extract FMT power for the
Oscillatory dynamics may be influenced by individual characteristics (i.e., age and clinical status). For this reason, we identified the frequency with maximal power for each subject in a window ranging 3.5 to $8 \mathrm{~Hz}$, and from 300 to $500 \mathrm{~ms}$ after FB onset (from the channels Fz \& FCz). Peak frequencies were close to the canonical Theta lower boundary $(4 \mathrm{~Hz})$ for the two groups alike $(\mathrm{HC}:$ mean $=4.20 \mathrm{~Hz}, \mathrm{SD}=$ 0.94; MDD: mean $=4.21 \mathrm{~Hz}, \mathrm{SD}=0.98$ ), thus we set the FMT frequency range from 3 to $7 \mathrm{~Hz}$ in all subsequent analyses, for both groups. For these reasons, FMT power changes $(3-7 \mathrm{~Hz})$ were defined as the mean computed within 0 to $200 \mathrm{~ms}$ and 300 to $500 \mathrm{~ms}$ after the response or FB respectively, and across channels Fz and FCz.

We further divided FMT power in the induced (non-phaselocked) and evoked (phase-locked) components to isolate oscillatory dynamics from time/frequency changes driven by ERPs. For this, we first computed the individual ERPs for each condition, time-locked to the response or the FB event. Second, the conditional ERP was subtracted from each single EEG epoch belonging to the relative condition. Third, the convolution and normalization procedure described above was repeated to obtain the induced FMT. The evoked power was derived by subtracting the induced from the total power (Cohen, 2014).

b

Feedback

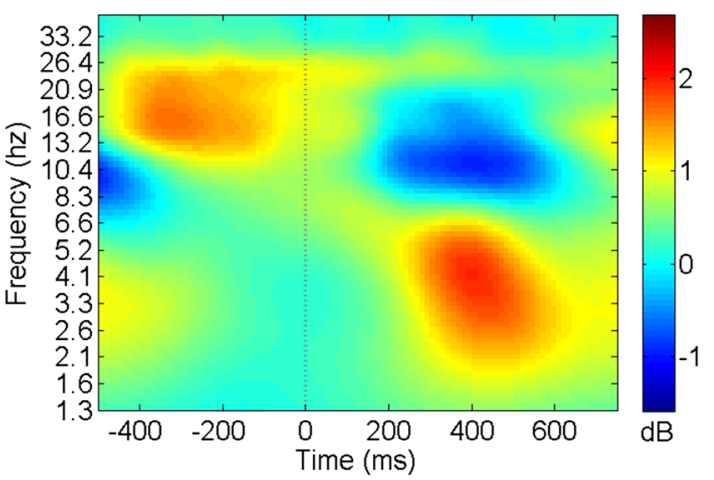

d
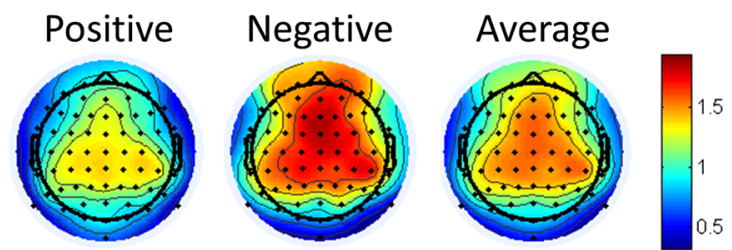

FB. (c) Horizontal scalp topographies of FMT power for the response (0$200 \mathrm{~ms}$ ), showing a clear FMT increase (when collapsing the three reward probabilities) at prefrontal electrodes along the midline ( $\mathrm{Fz} \& \mathrm{FCz})$ for incorrect compared with correct responses. (d) Horizontal scalp topographies of FMT power for the FB (300-500 ms), showing a clear FMT increase (when collapsing the three reward probabilities) at prefrontal electrodes along the midline ( $\mathrm{Fz} \& \mathrm{FCz}$ ) for negative (incorrect) compared with positive (correct) feedback 


\section{Frontal alpha-asymmetry}

All cleaned FB-locked epochs were included in this analysis, merging reward probability and accuracy factors. Whereas frontal alpha asymmetry is often computed using resting state EEG recordings, we analyzed it using active task data, because it has been shown that emotionally or motivationally relevant states may produce more robust individual differences than resting state data (i.e., response capability model, see Allen \& Reznik, 2015; Coan et al., 2006). Using this framework, MDD impairments in approach-motivation may emerge as a lateralized state response while approaching the FB. The segmented EEG data were converted to the scalp Laplacian (Kayser $\&$ Tenke, 2006), a reference-free current sources density estimation, to increase spatial selectivity and to minimize volume conduction. Because the Laplacian attenuates the contribution of distal volume-conducted sources (e.g., the occipital cortex and deep sources), it highlights the contribution of local electrode activities and radial dipoles (Perrin, Pernier, Bertrand, \& Echallier, 1989; Smith, Reznik, et al., 2017), thus improving the topographical localization of surface EEG signals. We computed the power spectral density (PSD) applying a fast Fourier transform (FFT) on the task data (spectopo() function), obtaining a $\mathrm{dB}$ converted estimation of relative power in a range of frequencies, with unit $10 * \log _{10}\left(\mathrm{uV}^{2} / \mathrm{Hz}\right)$. The FFT transform was applied to each epoch in a single 1second segment ( -100 to $900 \mathrm{~ms}$ relative to the $\mathrm{FB}$ ) weighted with a Hamming window (512-point window length given a sampling rate of $512 \mathrm{~Hz}$ ). The resulting PSD values were averaged across epochs for each subject and channel. Alpha power was defined as the average in the $8-13-\mathrm{Hz}$ range.

We further adopted a stringent standardization procedure that controls for individual variability in the bandpower estimation. For each subject, normalized singlesite Alpha power values were computed by dividing the power at each channel by the summed power across all channels; then, these ratios were transformed in $\mathrm{Z}$ scores, normalizing over all electrodes (Smith, Reznik, et al., 2017). This procedure allows to control for individual nuisance variable, such as scalp thickness and overall global power, providing a metric suited for exploring each homologous site's contribution to the lateralization, as well as correlations with criterion variables (e.g., clinical scales).

\section{Statistical analyses}

At the behavioral level, learning was expressed as percentage of correct responses varying as a function of time, using four consecutive bins of trials ( Bakic et al., 2017).
We compared the learning performance between MDD patients and HCs by means of a mixed-design ANOVA with reward probability and bin as within-subject factors, as well as group as between-subject factor. We also analyzed the effects of group and reward probability on reaction times (RT) for correct responses, as well as the amount of "too late" responses, by means of mixeddesign ANOVAs.

At the electrophysiological level, we analyzed FMT power changes at the response and FB levels separately, and we compared MDD patients to HCs by means of a mixeddesign ANOVA with accuracy and reward probability as within-subject factor, as well as group as between-subjects factor. Follow-up statistical analyses on the evolution of FMT power across successive trials were performed using Bayesian Multilevel Models (BMLM), implemented in R ( R Core Team, 2017) with the "brms" package (Bürkner, 2017; Nalborczyk, Batailler, Loevenbruck, Vilain, \& Bürkner, in press).

Alpha asymmetry was assessed considering the normalized Alpha power at typical frontal sites (F4 \& F3). We included in the analysis parietal sites (P4 \& P3) to establish the specificity of the effect for the frontal region. We compared frontal Alpha asymmetry for MDD patients to HC by means of a mixed-design ANOVA with region (frontal or parietal) and hemisphere (right or left) as within-subject factor and group as between-subjects factor. To assess the spatial localization of the frontal alpha asymmetry effect found with the first analysis, we performed a second analysis where we used an extended array of frontal homologous pairs (F2 \& F1, F4 \& F3, F6 \& F5, F8 \& F7). For this analysis, we used a mixeddesign ANOVA with pair and hemisphere as withinsubject factor and group as between-subjects factor. Last, we assessed the reliability of task-related Alpha asymmetry by means of split-half correlations. For either the HC or MDD group, we split the dataset according to odd and even trials (accuracy and probability conditions being balanced) and computed asymmetry scores between a set of frontal and parietal sites (F2-F1, F4-F3, F6-F5, F8-F7, P4-P3). Based on the raw Alpha power (without normalization), the asymmetry score was defined as the difference between the right-site and the left-site PSD (i.e., $10 * \log _{10}[$ Right $]-10 * \log _{10}[$ Left $]$ ), with higher values on this index putatively reflecting relatively greater left activity (i.e., relatively greater right alpha). Pearson's product-moment correlation coefficients were calculated between asymmetry scores derived by either odd or even trials, for each location and group.

For all the analyses, the Greenhouse-Geisser procedure was adopted to correct the degrees of freedom when the sphericity was violated. For post-hoc pairwise comparisons, a Bonferroni correction was used. 


\section{Results}

\section{Clinical and behavioral data}

As shown in Table 1, MDD patients had significantly higher depression scores (on all scales used) than HCs at baseline. Behavioral task data confirmed that for HCs, learning was influenced by time and reward probability, as expected (Bakic et al., 2014; Eppinger et al., 2008). More specifically, learning was steep and the highest for the deterministic condition, intermediate for the probabilistic condition, and absent for the random one. MDD patients exhibited the same learning profile (Fig. 2). Comparing MDD patients with HCs, the ANOVA failed to evidence a significant group $\mathrm{x}$ reward probability $\mathrm{x}$ bin $[F(4.59,303.21)=0.327, p=0.883, \eta 2 p=0.005]$ or group $\mathrm{x}$ reward probability interaction $[F(2,132)=0.297, p=$ $0.744, \eta 2 \mathrm{p}=0.004]$, or main effect of group $[F(1,66)=$ $0.771, p=0.383, \eta 2 p=0.012]$, whereas the reward probability $\mathrm{x}$ bin interaction was highly significant $[F(4.59,303.21)=29.229, p<0.001, \eta 2 p=0.307]$ and unambiguously translated improved behavioral performance across time when reward probability increased, for both groups. The analysis for RT speed showed significant main effects of group $[\mathrm{F}(1,66)=6.632, p=0.012, \eta 2 p=$ $0.091]$ and reward probability $[\mathrm{F}(2,132)=7.511, p<$ $0.001, \eta 2 p=0.102]$, indicating overall slower responses for MDD patients than HCs, as well as faster RTs when reward probability increased (Fig. 3b). For each condition, the number of "too late" responses was modest, yet larger for MDD patients (mean $=4.10, \mathrm{SE}=0.36$ ) than $\mathrm{HCs}$ $($ mean $=2.96, \mathrm{SE}=0.24)[\mathrm{F}(1,66)=6.971, p=0.010$, $\eta 2 \mathrm{p}=0.096]$, and varied across the three reward probability conditions $[\mathrm{F}(1.83,121.06)=7.981, p<0.001, \eta 2 \mathrm{p}=$ $0.106]$, increasing when reward probability decreased. We also used computational modeling to extract alternative indices of learning, including the learning rate and an exploration parameter (Jepma \& Nieuwenhuis, 2011), but failed to observe group differences for them. A significantly lower amount of switches after negative FB for MDD patients compared with HCs was observed only during the second part of the experiment (bins 3 and 4; see Bakic et al., 2017 for details regarding these analyses).

\section{Fronto-midline Theta}

As shown in Fig. 4, most of the total FMT power reflected the modulation of ongoing theta-band oscillations that occurred during the response or the FB but was not phase-locked to them (i.e., induced). Thus, we focused our analyses on the induced FMT only, that is the time-frequency representation in the Theta band of EEG dynamics that are task-related (i.e.,
Table 1 Demographic and clinical data for HCs and MDD patients (means are provided together with the standard deviations in parenthesis). Independent samples $t$-tests for BDI II $(\mathrm{df}=64)$, Anhedonia subscale of BDI II ( $\mathrm{df}=64)$, HAM D ( $\mathrm{df}=66$ ), SHAPS ( $\mathrm{df}$ $=66)$, and TEPS $(\mathrm{df}=66)$, with the corresponding subscales $(\mathrm{dfs}=66)$. Note that due to some missing data, the degrees of freedom (df) were different for the BDI II scale. *Corresponds to $p<0.05$, whereas $* *$ to $p<$ 0.01

\begin{tabular}{llll}
\hline & HC & MDD & $\mathrm{t}$ \\
\hline Number & 34 & 34 & \\
Gender (F/M) & $27 / 7$ & $27 / 7$ & \\
Age & $36.21(11.66)$ & $42.68(11.69)$ & $-2.29^{*}$ \\
BDI_II & $4.26(4.39)$ & $31.81(9.23)$ & $-15.63^{* *}$ \\
$\quad$ Anhedonia & $0.76(1.05)$ & $5.13(2.15)$ & $-10.57^{* *}$ \\
HAM_D & $1,18(2,04)$ & $21.47(5,29)$ & $-20,87^{* *}$ \\
SHAPS & $0.59(2.41)$ & $7.21(4.10)$ & $-8.11^{* *}$ \\
TEPS & $79.12(8.34)$ & $59.45(13.22)$ & $7.34^{* *}$ \\
$\quad$ Consumatory & $37.62(5.11)$ & $29.37(7.37)$ & $5.36^{* *}$ \\
$\quad$ Anticipatory & $41.50(5.63)$ & $30.08(7.47)$ & $7.12^{* *}$ \\
\hline
\end{tabular}

${ }^{*} p<0.05 ; * * p<0.01$

relative to the prestimulus baseline) but do not contribute to ERPs. $^{2}$

Induced FMT oscillatory activity was analyzed separately at the response and FB levels to ascertain that reward probability influenced these two levels in opposite directions. Importantly, we assessed whether abnormal RL in MDD patients was evidenced by systematic changes in FMT power, depending on reward probability and the level at which this information was processed (either response or feedback level). More specifically, we expected a larger group difference at the FB level when reward probability was low compared with high, due to a deficient sustained exploration of the FB in MDD. At the response level, the main effect of rewardprobability was significant $[\mathrm{F}(2,132)=3.40, p=0.036, \eta 2 \mathrm{p}$ $=0.049]$, as well as the main effect of accuracy $[\mathrm{F}(1,66)=$ $26.42, p<0.001, \eta 2 p=0.286]$. These main effects were accounted for by a monotonic decrease of FMT power as a function of decreasing reward-probability and by higher power for incorrect compared with correct responses, for the two groups alike (Fig. 6a). Moreover, reward probability interacted with accuracy $[\mathrm{F}(2,132)=10.74, p<0.001, \eta 2 \mathrm{p}$ $=0.140]$, indicating that the monotonic power decrease along decreasing probabilities was evidenced for incorrect responses

\footnotetext{
${ }^{2}$ The choice of analyzing the induced component of FMT was not motivated by a different physiological interpretation for the induced vs. evoked component of the signal (see Donner and Siegel, 2011; Gray and Singer, 1989; Tallon-Baudry and Bertrand, 1999). Rather, it was based on a previous EEG study linking the induced FMT to behavioral adaptation (Cohen \& Donner, 2013), as well as our goal to supplement the standard ERP data analysis (presented elsewhere, see Bakic et al., 2017) with time-frequency decompositions for which the specific contribution of the evoked/ERP component was removed.
} 
a

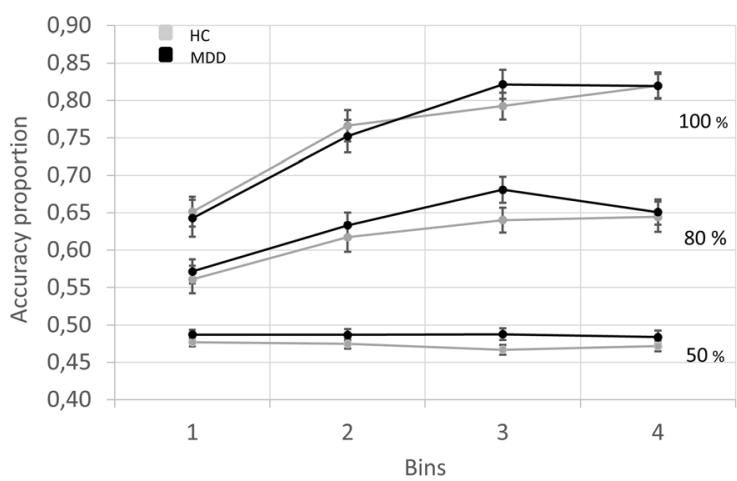

b

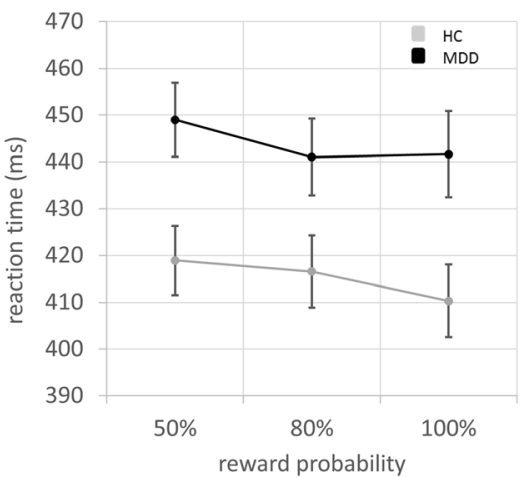

Fig. 3 Behavioral results. (a) Accuracy data (i.e., proportion of correct responses) decomposed as a function of bin, condition and group. Each bin corresponds to the average of 40 trials (20 consecutive trials per

only [linear contrast: $\mathrm{F}(1,66)=21.04, p<0.001, \eta 2 \mathrm{p}=0.242]$. For correct responses, FMT power followed the opposite trend [linear contrast: $\mathrm{F}(1,66)=4.00, p=0.050, \eta 2 p=0.057]$. In addition, FMT power differed between correct and incorrect responses only for the probabilistic $(80 \%)[\mathrm{F}(1,66)=9.41, p=$ $0.003, \eta 2 \mathrm{p}=0.125]$ and deterministic $(100 \%)[\mathrm{F}(1,66)=$ $35,60, p<0.001, \eta 2 p=0.350]$ conditions; this difference was not significant for the random $(50 \%)$ condition $[\mathrm{F}(1,66)$ $=0,19, p=0.663, \eta 2 p=0.072]$. Interestingly, this analysis also showed a significant interaction between group and accuracy $[\mathrm{F}(1,66)=6.35, p=0.014, \eta 2 p=0.088]$, indicating a clearer separation between correct and incorrect responses for HCs $[F(1,66)=29.34, p<0.001, \eta 2 p=0.308]$ than MDD patients $[\mathrm{F}(1,66)=3.43, p=0.068, \eta 2 \mathrm{p}=0.049]$, who in turn showed a trend for stronger FMT power after correct responses compared with $\mathrm{HCs}[\mathrm{F}(1,66)=3.38, p=0.070, \eta 2 \mathrm{p}$

a

Healthy controls

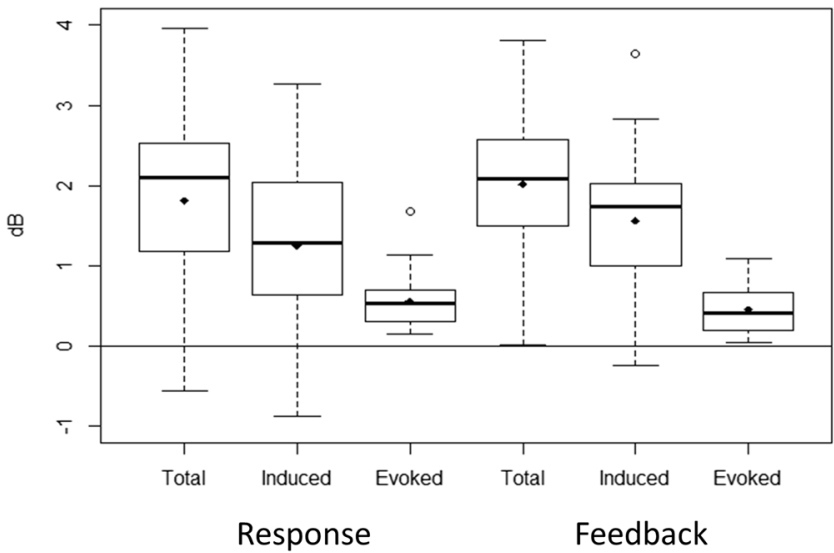

Fig. 4 (a) Boxplot analysis showing for each level separately (either response or FB), the proportion of total, induced and evoked FMT power changes for HCs. These FMT power changes correspond to the average of the two response accuracies and three probability conditions. The bold horizontal line represents the median, the box represents the interquartile range, and the whiskers extend to the last data point within 1.5 times the interquartile range. Additional solid black symbols indicate condition for each of the 2 task blocks). (b) Response latencies (for correct responses) decomposed as a function of group and reward probability. The error bar corresponds to 1 standard error of the mean

$=0.049]$. The main effect of group $[\mathrm{F}(1,66)=0.46, p=0.500$, $\eta 2 \mathrm{p}=0.007]$, interaction between group and reward probability $[\mathrm{F}(2,132)=0.09, p=0.918, \eta 2 \mathrm{p}=0.001]$, or the three-way interaction $[\mathrm{F}(2,132)=0.42, p=0.659, \eta 2 \mathrm{p}=0.006]$ were all nonsignificant. At the feedback level, the ANOVA showed significant main effects of accuracy $[\mathrm{F}(1,66)=18.79, p<$ $0.001, \eta 2 \mathrm{p}=0.222]$ and reward-probability $[\mathrm{F}(1.83,120.99)$ $=11.06, p<0.001, \eta 2 p=0.144]$. Negative FB elicited stronger FMT power than positive one, whereas a symmetric effect of reward probability (relative to the response level) was found: FMT power monotonically increased with decreasing reward-probability (Figs. 5 and 6). Unlike what we found at the response level, we did not observe a significant interaction between accuracy and reward probability $[\mathrm{F}(1.74,115.02)=$ $0.01, p=0.989, \eta 2 p=0.000]$ or between accuracy and group $[\mathrm{F}(1,66)=1.13, p=0.292, \eta 2 \mathrm{p}=0.017]$ at the feedback level.

b

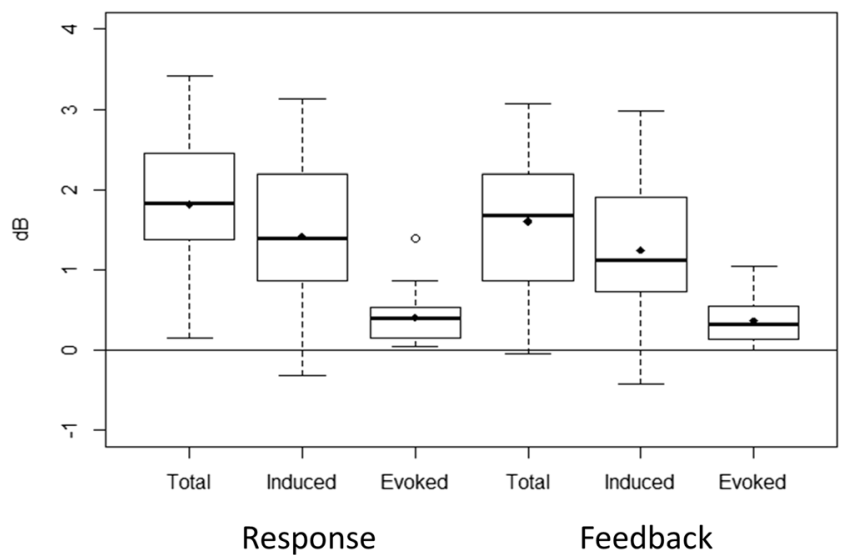

the mean. This analysis shows that irrespective of the level considered, the induced (non-phase-locked) component of FMT accounted for most of the total FMT. By comparison, the evoked FMT (phase-locked - captured by ERPs) reflected a much smaller portion. This difference indicates a larger contribution of non-phase-locked than phase-locked responses (ERPs) to FMT power after both response and FB. (b) The same pattern was seen in MDD patients 
a

Healthy controls
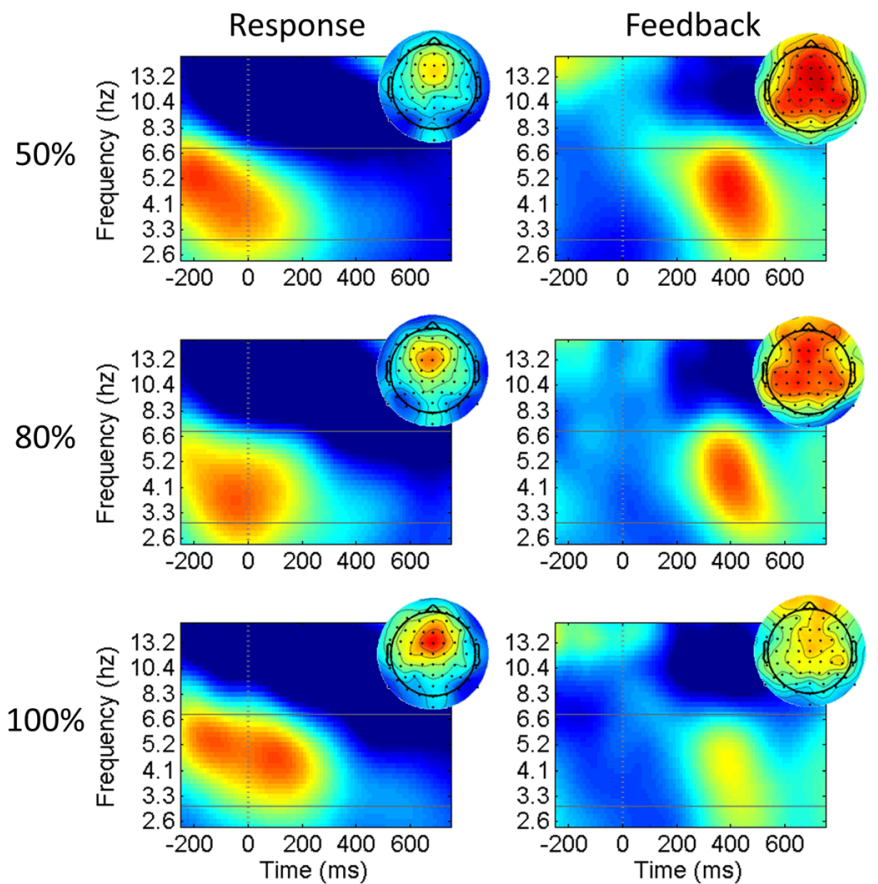

Fig. 5 (a) FMT (3-7 Hz) power at electrodes $\mathrm{Fz}$ and $\mathrm{FCz}$ (combined) for HCs $(n=34)$, separately for incorrect response ( $0-200 \mathrm{~ms}$ after its onset) and negative feedback (300-500 ms after its onset) and for each reward probability apart. Superimposed on each plot, the corresponding horizontal scalp topography is presented. (b) Same analysis for MDD patients $(\mathrm{n}=34)$. For both groups, FMT power varied with reward

The main effect of group approached significance $[\mathrm{F}(1,66)=$ $2.82, p=0.098, \eta 2 \mathrm{p}=0.041]$, reflecting a trend for a generally reduced FMT power across all conditions in MDD patients compared with HCs. Likewise, the interaction between group and reward probability was trend significant only $[\mathrm{F}(1.83,120.99)=2.37, p=0.102, \eta 2 \mathrm{p}=0.035]$. The three-

a

Response

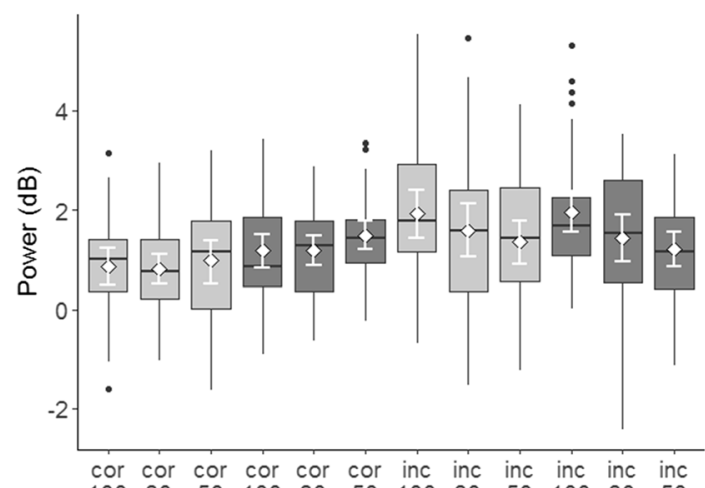

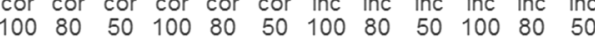

Fig. 6 The boxplots show FMT power $(3-7 \mathrm{~Hz}$ ) recorded at electrodes $\mathrm{Fz}$ and $\mathrm{FCz}$ (combined) separately for the response (a) and the FB (b) levels, and for each accuracy level and reward probability. The two groups are coded with different shades of grey. The horizontal line represents the median, the box represents the interquartile range, and the whiskers

\section{b MDD patients}
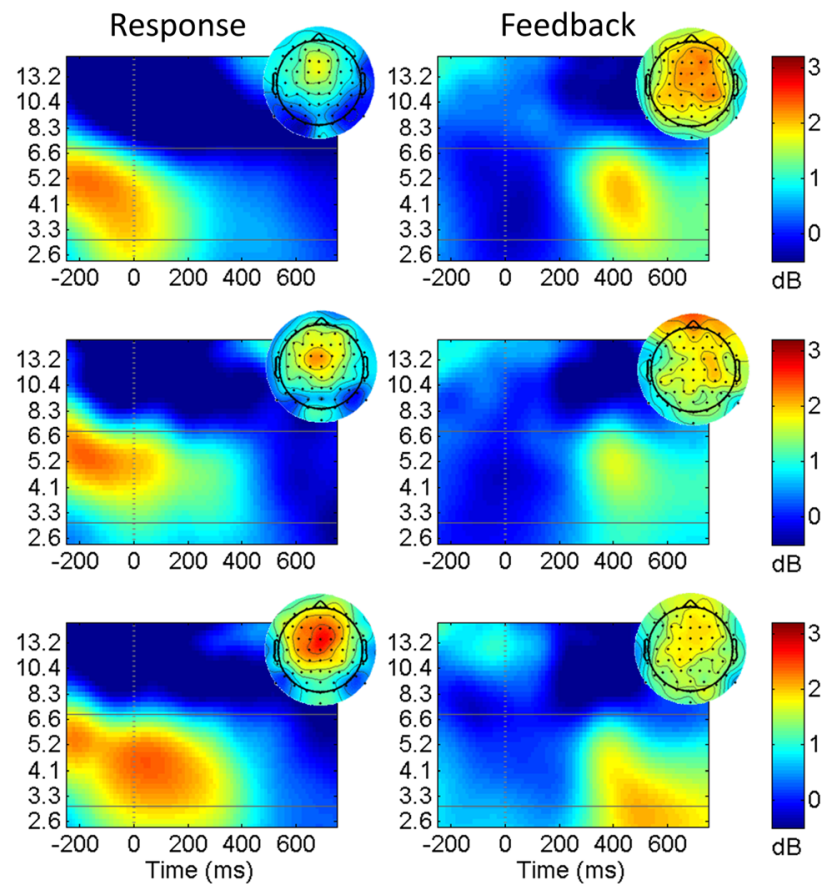

probability but in opposing directions for incorrect response and negative FB: it increased with increasing reward probability at the response level while showing the opposite effect at the FB level. At the FB level, FMT power was reduced for MDD patients compared to HCs, especially for the low reward probability condition

way interaction was not significant $[\mathrm{F}(1.74,115.02)=0.87, p$ $=0.407, \eta 2 \mathrm{p}=0.013]$.

To assess whether MDD patients showed a drop in motivation to decipher the most complex S-R associations (random condition) based on the feedback information, as the trend significant interaction between group and reward b

Feedback

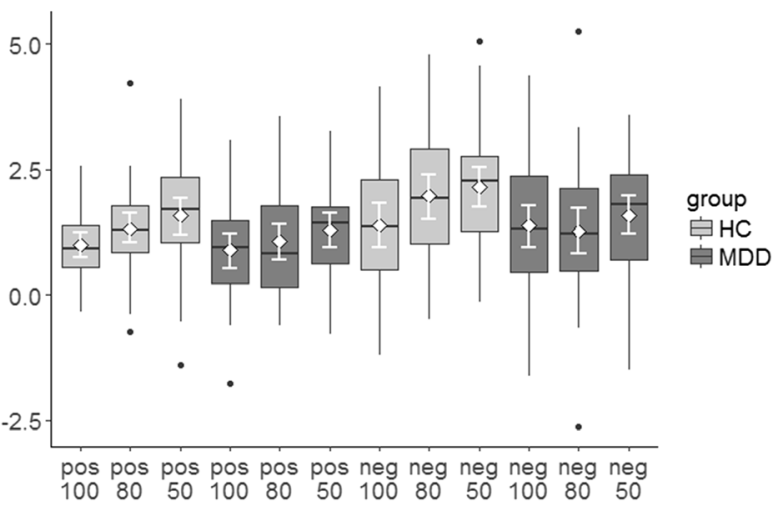

extend to the last data point within 1.5 times the interquartile range. The black points indicate the outliers. Superimposed in white, the diamond symbols indicate the mean and the extending ranges cover the $95 \%$ confidence intervals 
probability indirectly suggested (see above), we performed a follow-up analysis where we extracted FMT power changes at the single-trial level (random condition) and modeled their evolution across successive trials. We reasoned that if MDD patients showed a drop in motivation, then FMT power should decrease in a steeper manner across trials for them in this condition, relative to the HCs. Relying on a Bayesian multilevel model analysis, we assessed the amount of evidence in favor of this specific hypothesis. The methodological and statistical details of this single-trial analysis are provided in the Supplementary Materials section. Figure 7a shows the outcome of this analysis and is based on the model that best fit the observed data. This model included the main effects of time, accuracy, group, and their interactions (see Supplementary Materials). Based on this model, we examined the difference between the probability distributions of the conditions of interest. Statistical results showed that for positive FB, the hypothesis of a steeper decrease of FMT power across time for MDD patients than HCs was 4.1 times more likely than the alternative one, predicting an opposite effect. For negative FB, results showed that it was 34.7 times more likely that FMT power decreased across trials more sharply for MDD patients than HCs compared with the opposite hypothesis. Last, the hypothesis that the group difference in the

a

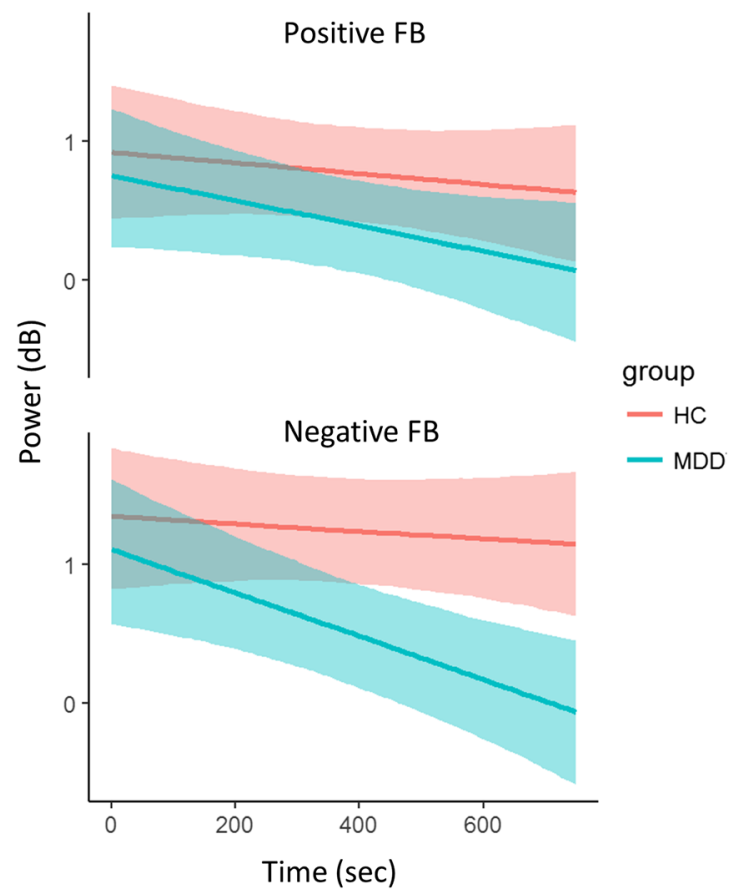

Fig. 7 Temporal evolution of FMT power (FB level) across consecutive trials, for the $50 \%$ (random) probability condition. (a) Results of the Bayesian multilevel modeling. The figure represents the populationlevel marginal effects of the predictors time, accuracy, and group on the estimated FMT power. These estimates are based on the model that best fit the observed data (see Supplementary Materials). The lines represent the mean of posterior probability samples at each second from the beginning of the task blocks and for each condition. The shading represent the steepness of the slope was larger for negative than positive FB was 3.2 times more likely than the opposite one. Thus, this single trial analysis provided strong evidence in favor of the hypothesis that FMT power for negative FB decreased more sharply across trials for MDD patients than HCs, as well as some evidence that this effect was larger for negative compared with positive FB.

\section{Frontal alpha-asymmetry}

To examine possible anomalies in approach motivation in MDD patients, we compared frontal alpha asymmetry (feedback level) between them and HCs. The ANOVA comparing frontal and parietal normalized Alpha power showed a significant two way interaction between hemisphere and group $[F(1,66)=4.90, p=0.030, \eta 2 \mathrm{p}=0.069]$. Post-hoc comparison revealed a significant effect of hemisphere for the MDD group only $[F(1,66)=4.84, p=0.031, \eta 2 p=0.068]$ translating a negative Alpha asymmetry index (left: mean $=0.103$, SE $=0.145$; right: mean $=-0.316, \mathrm{SE}=0.105)$. Importantly, this effect also was qualified by a significant interaction with region $[F(1,66)=4.63, p=0.035, \eta 2 p=0.066]$. Post-hoc comparisons revealed a significant effect of hemisphere for frontal sites in the MDD group exclusively $F(1,66)=5.56, p=0.021$,

\section{b}

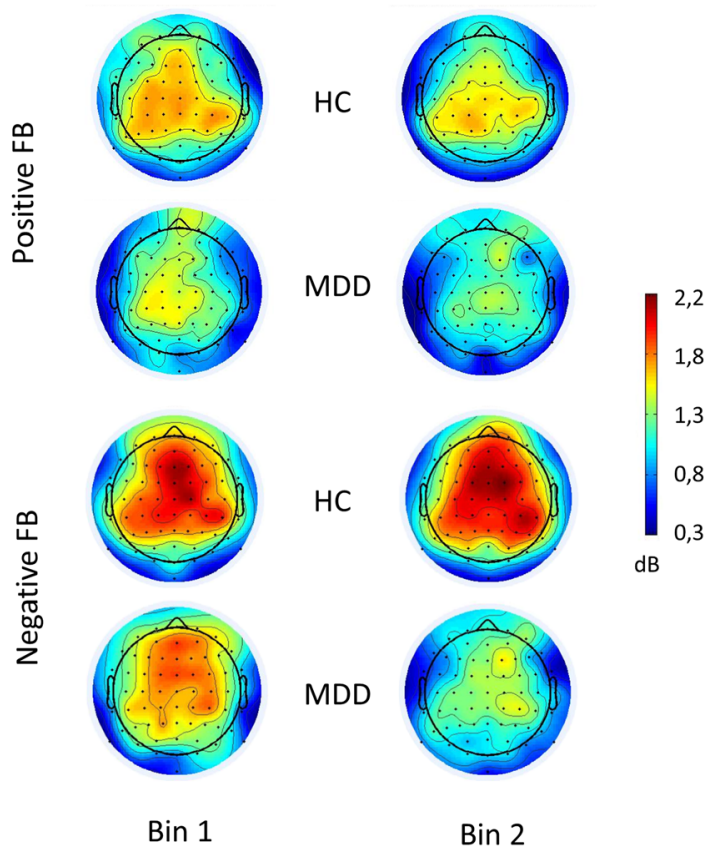

95\% credible interval around them. (b) For a comparison to the observed data, the horizontal scalp topographies show FMT power for the FB (300-500 ms), for each accuracy level and group. To represent roughly the effect of time, FMT power was computed separately for the first and second bin of trials, considering all trials available for each subject. This was done for each block separately, before FMT power for the two blocks was collapsed 
$\eta 2 p=0.078$, expressed as a negative asymmetry index (corresponding to relatively higher left than right alpha power, thus translating a relatively lower left than right frontal activation; left: mean $=0.343, \mathrm{SE}=0.220$; right: mean $=-0.345$, $\mathrm{SE}=0.163)$ (Fig. 8). With regard to the HC group, the effect of hemisphere did not reach significance, although showed the opposite trend at the frontal region (left mean $=-0.133, \mathrm{SE}=$ 0.220 , right mean $=0.260, \mathrm{SE}=0.163$ )

Moreover, in an additional analysis we considered an extended array of frontal electrodes on both sides (F2 \& F1, F4 \& F3, F6 \& F5, F8 \& F7) to assess whether frontal alpha asymmetry was circumscribed to a few isolated locations. The ANOVA comparing normalized Alpha power across frontal pairs showed a significant main effect of pair $[F(2.29,150.90)=50.79, p<0.001, \eta 2 p=0.435]$. This main effect was accounted for by a linear increase of Alpha power from medial to lateral pairs $[F(1,66)=94.21, p<0.001, \eta 2 p=$ 0.588]. Interestingly, the ANOVA showed also a significant three-way interaction between pair, hemisphere, and group $[F(2.01,132.51)=4.43, p=0.014, \eta 2 p=0.063]$. Post-hoc comparison revealed a significant effect of hemisphere in the MDD group and for the second pair selectively (F4 \& F3; F4: mean $=-0.345, \mathrm{SE}=0.163 ; \mathrm{F} 3$ : mean $=0.343, \mathrm{SE}=0.220$; $[F(1,66)=5.56, p=0.021, \eta 2 \mathrm{p}=0.078])$.
Finally, the split-half correlations indicated a strong reliability of Alpha asymmetry, translating a stable topographic distribution of Alpha power across different trials. For each site considered (F2-F1, F4-F3, F6-F5, F8-F7, P4-P3), the Alpha asymmetry score was highly correlated between odd and even trials, for both groups (HC range: $\mathrm{r}=0.987$ 0.997, $\mathrm{N}=34$; MDD range: $\mathrm{r}=0.933-0.995, \mathrm{~N}=34$ ).

Last, we also performed exploratory correlation analyses between the symptomatology or severity of depression and these electrophysiological measures, as well as between FMT and frontal Alpha power (see Supplementary Materials).

\section{Discussion}

Previous research in behavioral neuroscience, neuroimaging, and psychiatry demonstrated that dysfunctions in frontostriatal reward systems (i.e., Anhedonia, in combination with exaggerated stress responsiveness) play a central role in the etiology and maintenance of MDD (for a review, see Pizzagalli, 2014). Besides strong impairments in reward sensitivity (Bress et al., 2012; Foti, Carlson, Sauder, \& Proudfit, 2014; Weinberg, Liu, Hajcak, \& Shankman, 2015), abnormal reward anticipation and motivation are cardinal features of a

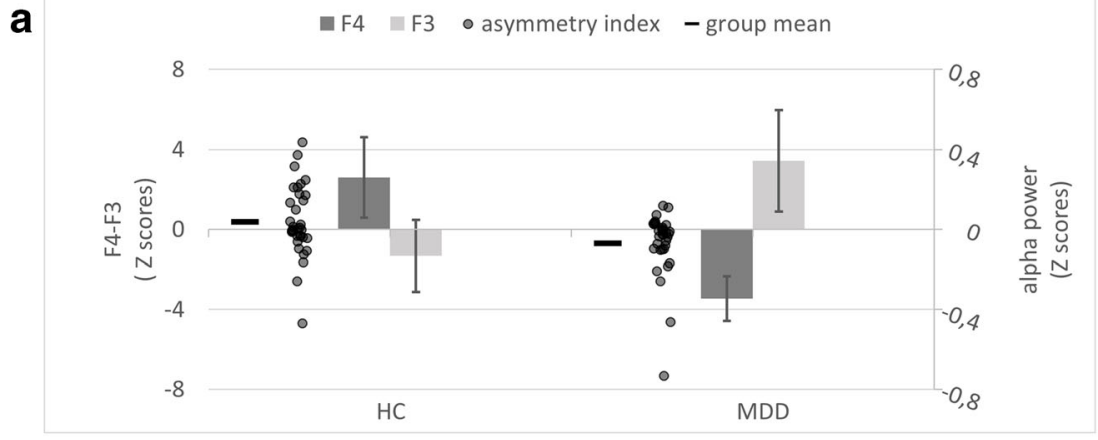

b

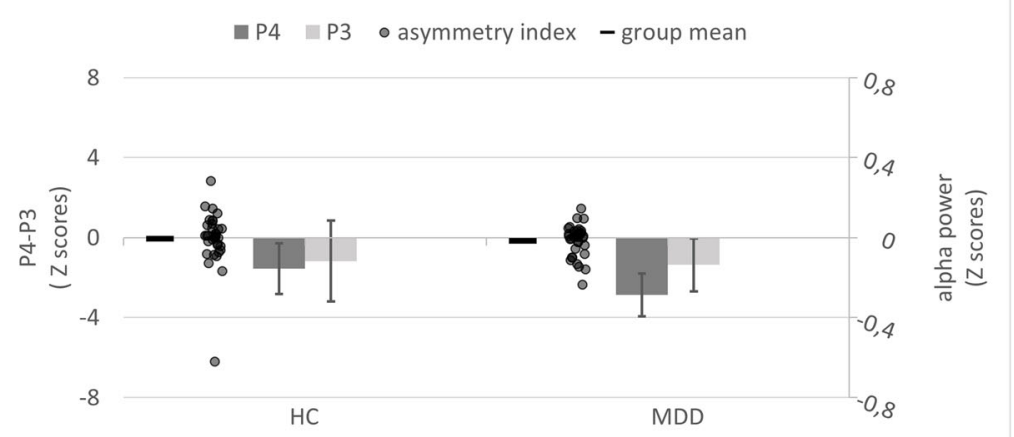

C

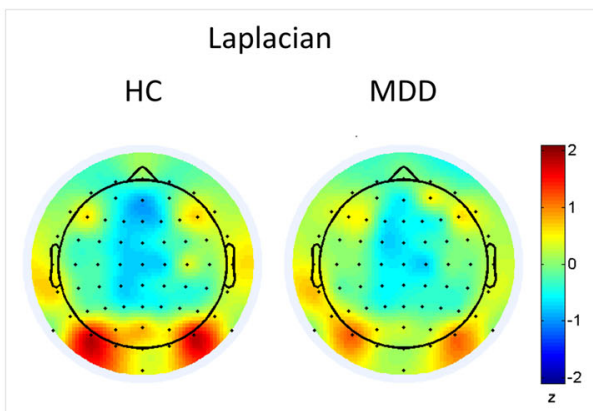

Original

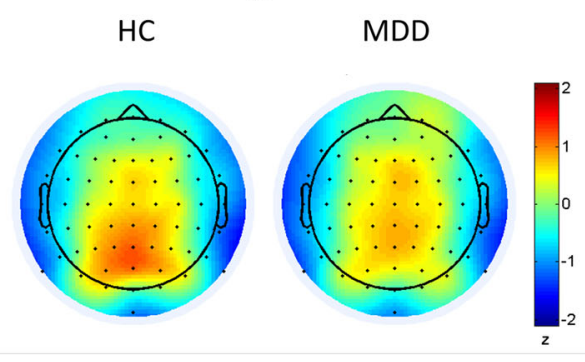

Fig. 8 (a) Frontal alpha asymmetry results, separately for HCs and MDD patients. (b) Parietal alpha asymmetry results for comparison purposes. Histograms represent mean alpha power for left (F3, P3) and right (F4, $\mathrm{P} 4)$ channels, whereas the horizontal line bar reflects the mean asymmetry score (for each group) computed as the right- minus left-channel difference. The dots represent the subject-specific asymmetry scores. The error bar corresponds to 1 standard error of the mean. Note that both asymmetry scores and the alpha power at single channels refer to alpha power (with original unit $10 * \log _{10}\left(\mathrm{uV}^{2} / \mathrm{Hz}\right)$ ) converted to $\mathrm{Z}$ scores by means of a within-subject topographical normalization. (c) Horizontal scalp topographies of alpha power (z scores), separately for HCs and MDD patients, computed on the Laplacian-filtered data (top) and the non-filtered data (bottom) 
anhedonia in MDD (i.e., "wanting") (Berridge \& Robinson, 2003; Thomsen, 2015; Treadway \& Zald, 2011), which in turn undermine the possibility to optimize behavior (learning) as a function of reward in these patients (Pizzagalli et al., 2008; Vrieze et al., 2013; Whitton et al., 2016). Such impairments should be visible during RL, where learning performance critically depends on the use, evaluation, and exploration of specific incentives. In the present study, we sought to lend additional support to this dominant framework by comparing the neurophysiological correlates of RL and approach-related motivation between MDD patients and matched HCs. Therefore, we tested a large cohort of treatment-resistant MDD patients (enrolled in a treatment study, see Duprat et al., 2016) and compared them to healthy, matched controls on a standard probabilistic learning task (Eppinger et al., 2008). We explored systematic changes of FMT oscillations as a function of reward probability, separately for the response (internal monitoring) and feedback level (external monitoring). FMT provides a reliable electrophysiological correlate of performance monitoring, putatively mediating the impact of RPE on behavioral adaptation and learning (Cavanagh et al., 2010; Cohen et al., 2008, 2011; E. H. Smith et al., 2015; van de Vijver et al., 2014). Interestingly, FMT has been proposed to signal the amount of control to be allocated over performance during extended and cognitive demanding tasks (Holroyd \& Umemoto, 2016), but very few studies to date have evaluated systematically whether MDD could influence it during RL (Cavanagh, Bismark, Frank, \& Allen, 2011). ${ }^{3}$ Moreover, to examine possible group differences in approach motivation, we also extracted hemispheric frontal alpha asymmetry, measured throughout the task as a state response, and using the most recent methodological recommendations for this metric, including Laplacian transformation and a stringent normalization procedure (Allen \& Reznik, 2015; E. E. Smith, Reznik, et al., 2017; Stewart et al., 2014).

The present results do not support the assumption that anhedonia in MDD entails impaired RL, because we failed to observe clear-cut deficits in RL at the behavioral and EEG (FMT) levels in a large sample of MDD patients characterized by high levels of anhedonia. However, these results show that MDD and anhedonia are accompanied by deficits in approach motivation, as suggested by frontal alpha asymmetry as well as

\footnotetext{
${ }_{3}^{3}$ Other studies already used in the past advanced time/frequency methods to evaluate FB processing in healthy and clinical populations, yet focusing on the phase-locked component of the EEG signal mostly (i.e., extracting power changes in specific bands after epochs averaging) in an attempt to parse the differential contribution of overlapping ERP components to the ERP power spectrum (Bernat, Nelson, \& Baskin-Sommers, 2015; Bernat, Nelson, Steele, Gehring, \& Patrick, 2011; Foti, Weinberg, Bernat, \& Proudfit, 2015). Here, we used a very different approach and data analysis, where we purposely removed the ERP activity from the original EEG signal and used a time-frequency decomposition performed at the single trial level (Cohen, 2014; Cohen \& Donner, 2013) with the aim to explore the contribution of non-phase-locked activity to power changes (in the theta band) as a function of reward probability and MDD.
}

by a steep FMT power decrease across successive trials when considering the most challenging RL condition. In fact, despite being classified as at least stage I treatment resistant (Fava, 2003) and showing a high depression's severity as well as clear Anhedonia (both consummatory and anticipatory; Table 1), these patients actually showed globally spared RL processes (Fig. 3a). Learning was titrated at the behavioral level using either standard accuracy measures (Bakic et al., 2014; Eppinger et al., 2008) or alternative indices deriving from computational modeling, such as learning rate or exploration (Bakic et al., 2017). The two groups showed comparable RL-based effects for these different measures. The only exception was the rate of switches after negative FB, which was significantly lower for these MDD patients compared with the HCs during the second part of the experiment (bins 3 and 4), selectively (Bakic et al., 2017). This result suggested indirectly a possible drop in motivation and exploration across time in these MDD patients.

At the EEG level, FMT power was higher for incorrect than correct responses, and for negative than positive FB, as previously reported (Cavanagh, Figueroa, et al., 2012; Cavanagh et al., 2010; van de Vijver et al., 2014). As expected (van de Vijver et al., 2014), FMT power modulation strongly depended on reward probability and was symmetrical between incorrect responses and negative FB (Figs. 5 and 6). When the S-R was deterministic, FMT power was the largest for incorrect response. Conversely, when the S-R was random, FMT power was the largest for negative FB, confirming the sensitivity of this neurophysiological signal to reward-based learning. This neurophysiological effect aligns with the behavioral results showing that RL varied with reward probability. When learning was easy (deterministic S-R association), participants likely processed response errors at the response level on most trials, without the need to rely on the subsequent feedback to infer accuracy. By comparison, when it was hard or even impossible (probabilistic and random S-R associations, respectively), participants had to use actively the evaluative FB to infer accuracy, whereas evidence accumulated at the response level was probably too weak or absent. Hence, the corresponding effects on FMT power captured prediction errors and/or enhanced cognitive control in accordance with $\mathrm{RL}$ dynamics. Interestingly, only response errors, but not correct responses, elicited a large FMT power that decreased systematically with decreasing reward probability. At the FB level, both positive and negative FB showed a symmetric pattern compared to the response level, suggesting that FMT may reflect an unsigned prediction error signal. In fact, according to some authors (Cavanagh, Figueroa, et al., 2012; Hajihosseini \& Holroyd, 2013), FMT cannot reflect an axiomatic RPE coded by dopamine neurons, because it does not show an interactive effect between reward and expectancy (Caplin and Dean, 2008). Rather, it is mainly modulated by the (un)predictability of events in general, and it could reflect the amount of effort or control to be exerted as a result 
(output) of information processed by the ACC (including RPE signals), where the subjective value of the task might be estimated (Holroyd and Umemoto, 2016; Smith et al., 2015). In this scenario, the symmetric change in FMT power seen in our study between the response and FB levels across the three reward probability conditions could be explained by explicit predictions about performance (model-based reward learning; Dayan \& Berridge, 2014), being initially made and eventually violated: if the S-R association was deterministic, on most trials a positive prediction could readily be computed at the response level, and be violated in case of response error. Instead, if the S$\mathrm{R}$ association was probabilistic or random, the evaluative FB provided after the choice was respectively the main or only cue to gauge violations of prediction (in either direction).

Intriguingly, these effects were generally spared in MDD, disconfirming one of our main hypotheses. However, FMT power was slightly different between the two groups. At the response level, MDD patients showed only smaller differences in FMT power between correct and incorrect responses compared with HCs (Fig. 6a; Suppl. Fig. 1). Specifically, compared with HCs, MDD patients showed an overall increase of FMT for correct responses, which may translate increased uncertainty at the response level (i.e., increased response conflict). When considering the FB level, both HCs and MDDs showed a symmetric pattern in FMT power modulation as a function of reward probability relative to the response level. Interestingly, MDD patients showed a numerically blunted FMT power modulation at the FB level, especially when reward probability was low (and hence the hidden S-R mapping was hard to discover), although we failed to evidence a significant interaction effect between group and reward probability. Crucially, robust evidence for a group difference in this condition was provided by a follow-up analysis where we could model the evolution of FMT power across successive trials. As shown in Fig. 7, this group difference was expressed at the FB level in terms of a steeper decrease (slope) of FMT power as a function of time for MDD patients compared with HCs and not simply as impaired discrimination of the evaluative FB as being positive or negative (i.e., both groups showed a different intercept at time 0; see also Suppl. Fig. 5). Furthermore, this decrease of FMT power across successive trials was larger for negative compared with positive FB. These results suggest that both groups showed strong FMT power activity at the beginning of the task, but unlike MDD patients, HCs maintained enhanced cognitive control across time in response to $\mathrm{FB}$, despite its low reward value in this condition. To note, in this condition learning was made impossible by design. Consequently, this drop shown by MDD patients at the neurophysiological level could not be accompanied by an impaired behavioral performance, relative to the HCs. As such, these FMT results corroborate to some degree the assumption that MDD likely interferes with specific motivation processes active during reward-based learning, as if it impaired selectively the involvement of extra efforts or resources necessary to yield learning in a complex situation where stimuli and responses carry low reward values (Pizzagalli et al., 2005; Salamone, Correa, Farrar, \& Mingote, 2007; Thomsen, 2015; Treadway et al., 2012).

When considering specific motivation processes reflected by frontal Alpha asymmetry (as measured throughout the task as a state response to the FB; Fig. 8), the results were clearer and showed a negative frontal Alpha asymmetry for MDD patients only, when considering the F3-F4 pair selectively. This asymmetry was expressed by positive normalized Alpha power for the left frontal site (F3), but negative Alpha power for the right frontal site (F4), relative to the average Alpha activity measured across the entire scalp. By comparison, HCs did not show this asymmetry but actually an opposite pattern. This clear group difference in lateralized frontal activity is consistent with the assumption of abnormal approachrelated motivation in MDD (Eddie Harmon-Jones \& Gable, 2017; Nelson et al., 2017; Pizzagalli et al., 2005), expressed here as a motivational disengagement during FB presentation. Importantly, this effect was significant at frontal sites only, confirming a clear regional specificity. Moreover, this stateresponse metric of cortical activity was shown to be reliable and highly consistent across trials, for any site considered.

The observation of globally preserved reward-based learning at the behavioral (and FMT) level in MDD in our study is actually in line with some previous results reported in the literature showing normal learning performance during standard RL tasks with this emotional disorder (Cavanagh et al., 2011; Kunisato et al., 2012). To explain this result, three methodological elements are worth considering in the present case. First, we used a probabilistic learning task (Eppinger et al., 2008; Frank et al., 2005) based on "explicit" RL. Instructions clearly emphasized that the task was precisely about discovering different hidden S-R associations across successive trials and that reward delivery directly depended on the ability to do so. By comparison, other studies (Pizzagalli et al., 2008; Whitton et al., 2016) that reported impaired RL in MDD at the behavioral and neural levels usually used "implicit" task and reinforcement. In these cases, reward was used to promote an implicit response bias (i.e., conditioning), while its delivery was actually decoupled from the task instructions. As a result, different learning mechanisms are probably involved in these two situations (Berridge \& Robinson, 2003), and MDD might influence one of them only or more strongly than the other (i.e., when an implicit learning task is used primarily to promote reward-based learning). Second, behavioral impairments during RL found in MDD might actually depend not only on the type of RL task used, but also the nature of the reinforcer used to foster learning. We used so-called "primary" reinforcers (correct vs. incorrect response, hence related to selfefficacy), whereas behavioral impairments seen in MDD patients during RL in previous studies (see above) were usually 
observed when "secondary" reinforcers, such as small monetary reward, were used. Third, we cannot rule out the possibility that this discrepancy between the present and some previous studies might be explained by the patients' characteristics to some extent. Although our sample of MDD patients was relatively large and homogenous (Table 1), yet these patients were treatment-resistant, severely anhedonic, and hence not immediately comparable to MDD patients tested in earlier studies where different inclusion criteria were used (Cavanagh et al., 2011; Pizzagalli et al., 2008; Treadway et al., 2012). In this context, it is conceivable that their treatment resistance, combined with the fact that they were enrolled in a treatment study, may have artificially boosted specific motivation processes (such as their engagement in the task and willingness to perform well), eventually explaining why we failed to reveal clear deficits at the behavioral level during RL in these patients using this specific probabilistic learning task.

Our results suggest that impaired RL might not be a core feature of unipolar major depression and anhedonia. Accordingly, they align with recent neuroscientific evidence indicating that this mood disorder does not impair the main expression of dopaminergic-related RPE signals (Rutledge et al., 2017), which underpin RL. In comparison, the abnormal frontal Alpha asymmetry found in these MDD patients could reflect motivational deficits, in agreement with many earlier studies and models available in the extant literature (Allen, Urry, Hitt, \& Coan, 2004; Coan \& Allen, 2004; Davidson, 1998b, 2004; E Harmon-Jones \& Allen, 1997). Together, our new findings suggest the existence of two dissociable brain systems supporting RL: a cognitively driven approach-motivation system, which is probably impaired in MDD, and a corticostriatal dopaminergic reward network, which can be globally spared in this specific mood disorder. However, additional empirical work is needed to corroborate this conclusion, preferably using imaging methods, such as fMRI (in combination with EEG), which is appropriate to determine the respective contribution at the anatomical level of these two nonoverlapping brain networks to RL, as well as their differential vulnerability to MDD.

Although the current results await replication in new samples of MDD patients, they also have indirect clinical implications. In light of this dissociation outlined above, we surmise that therapies targeting a restoration of frontal lobe functioning in treatment resistant MDD patients, such as TMS (Fox, Buckner, White, Greicius, \& Pascual-Leone, 2012) or the combination of neurostimulation with cognitive control training for example (De Raedt, Vanderhasselt, \& Baeken, 2015), as well as interventions that may alter indirectly EEG asymmetry by improving motivation, such as cognitive behavior therapy (Moscovitch et al., 2011), might all help to improve approach motivation in the first place and subsequently counteract a drop in the sustained exploration of low reward cues in the environment. Accordingly, it would be valuable in future studies to compare RL using the same electrophysiological components as used here (i.e., FMT and frontal alpha asymmetry) before and after treatment or psychotherapy.

Last, at the methodological level, our study also adds to the existing EEG literature on RL by showing the added value of a careful exploration and modelling of FMT power changes across successive trials. Clear and compelling group differences emerged in the random condition when we examined the evolution of FMT power across time, unlike standard averages where they were less visible. These differences suggested indirectly that MDD patients failed to maintain a high level of cognitive control throughout the experiment when RL was challenging, which is consistent with a motivational impairment in these patients. We believe that this methodological approach is valuable, because a careful analysis of the evolution of FMT power changes across successive trials can reveal the temporal dynamic of RL and its modulation by MDD. Moreover, the use of a Bayesian multilevel modelling allows to deal with these (noisy) single-trial data, as well as to quantify the evidence for a given hypothesis in terms of probability.

\section{Conclusions}

The results of this study suggest that RL can be globally spared in MDD at the behavioral level. At the electrophysiological level, we found that FMT power substantially changed as a function of reward probability (thereby paralleling the behavioral results) and in accordance with the evidence available: while it augmented with increasing reward probability at the response level (internal monitoring), the reverse effect was found at the feedback level (exploration), suggesting a flexible engagement of this neurophysiological signal to optimize learning. These neurophysiological effects were similar for MDD patients and HCs in our study. However, when we examined FMT power changes at the single trial level when RL was challenging (i.e., reward probability was at chance level), MDD patients showed a steeper decrease across time than HCs, suggesting indirectly a drop in the ability to maintain a high level of cognitive control throughout the experiment in this condition, and hence the presence of a specific motivational deficit in these patients. Moreover, when focusing on frontal Alpha power, computed as a global state measure, or response capability throughout the experimental session, clear group differences emerged as well. More specifically, MDD was associated with a larger inhibition of the left prefrontal cortex that yielded a pronounced frontal Alpha asymmetry compared with HCs, confirming a general deficit in approach motivation in these patients (Coan \& Allen, 2004; Davidson, 1998b). The present study helps to clarify the neurophysiological mechanisms of RL and approach motivation and suggests that MDD can alter the latter while leaving the former globally spared. 
Acknowledgments The authors thank Ivan Grahek for the guidance in performing and reporting the BMLM statistical analysis and Ladislas Nalborczyk for further support with its implementation.

Funding RDR, $\mathrm{CB}$, and GP are funded by a Concerted Research Action Grant from Ghent University. GP is supported by a 2015 NARSAD Independent Investigator Grant from the Brain \& Behavior Research Foundation and by the Research Foundation Flanders (FWO, grant number 3G024716).

\section{Compliance with ethical standards}

Conflict of interest The authors declare that they have no conflict of interest.

Ethical approval All procedures performed in studies involving human participants were in accordance with the ethical standards of the institutional and/or national research committee and with the 1964 Helsinki declaration and its later amendments or comparable ethical standards.

Informed consent Informed consent was obtained from all individual participants included in the study.

Publisher's note Springer Nature remains neutral with regard to jurisdictional claims in published maps and institutional affiliations.

\section{References}

Admon, R., \& Pizzagalli, D. A. (2015). Dysfunctional reward processing in depression. Current Opinion in Psychology, 4, 114-118. https:// doi.org/10.1016/j.copsyc.2014.12.011

Allen, J. J. B., \& Reznik, S. J. (2015). Frontal EEG asymmetry as a promising marker of depression vulnerability: Summary and methodological considerations. Current Opinion in Psychology, 4, 9397. https://doi.org/10.1016/j.copsyc.2014.12.017

Allen, J. J. B., Urry, H. L., Hitt, S. K., \& Coan, J. A. (2004). The stability of resting frontal electroencephalographic asymmetry in depression. Psychophysiology, 41(2), 269-280. https://doi.org/10.1111/j.14698986.2003.00149.x

Bakic, J., Jepma, M., De Raedt, R., \& Pourtois, G. (2014). Effects of positive mood on probabilistic learning: Behavioral and electrophysiological correlates. Biological Psychology, 103, 223-232. https://doi.org/10.1016/j.biopsycho.2014.09.012

Bakic, J., Pourtois, G., Jepma, M., Duprat, R., De Raedt, R., \& Baeken, C. (2017). Spared internal but impaired external reward prediction error signals in major depressive disorder during reinforcement learning. Depression and Anxiety, 34(1), 89-96. https://doi.org/10. 1002/da.22576

Barch, D. M., Carter, C. S., Gold, J. M., Johnson, S. L., Kring, A. M., MacDonald, A. W., ... Strauss, M. E. (2017). Explicit and implicit reinforcement learning across the psychosis spectrum. Journal of Abnormal Psychology, 126(5), 694-711. https://doi.org/10.1037/ abn0000259

Beck, A., Steer, R., \& Brown, G. (1996). Beck Depression Inventory-II (BDI-II). Beck Depression Inventory-II. Retrieved from http://www. pearsonclinical.com/psychology/products/100000159/beckdepression-inventoryii-bdi-ii.html?Pid=015-8018-370

Bernat, E. M., Nelson, L. D., \& Baskin-Sommers, A. R. (2015). Timefrequency theta and delta measures index separable components of feedback processing in a gambling task. Psychophysiology, 52(5), 626-637. https://doi.org/10.1111/psyp. 12390
Bernat, E. M., Nelson, L. D., Steele, V. R., Gehring, W. J., \& Patrick, C. J. (2011). Externalizing psychopathology and gain/loss feedback in a simulated gambling task: Dissociable components of brain response revealed by time-frequency analysis. Journal of Abnormal Psychology, 120(2), 352-364. https://doi.org/10.1037/a0022124

Berridge, K. C., \& Robinson, T. E. (2003). Parsing reward. Trends in Neurosciences, 26(9), 507-513. https://doi.org/10.1016/S01662236(03)00233-9

Berridge, K. C., Robinson, T. E., \& Aldridge, J. W. (2009). Dissecting components of reward: "liking", "wanting", and learning. Current Opinion in Pharmacology, 9(1), 65-73. https://doi.org/10.1016/j. coph.2008.12.014

Bress, J. N., Smith, E. E., Foti, D., Klein, D. N., \& Hajcak, G. (2012). Neural response to reward and depressive symptoms in late childhood to early adolescence. Biological Psychology, 89(1), 156-162. https://doi.org/10.1016/j.biopsycho.2011.10.004

Bürkner, P.-C. (2017). brms : An R Package for Bayesian Multilevel Models Using Stan. Journal of Statistical Software, 80(1), 1-28. https://doi.org/10.18637/jss.v080.i01

Caplin, A., \& Dean, M. (2008). Axiomatic methods, dopamine and reward prediction error. Current Opinion in Neurobiology, 18(2), $197-$ 202. https://doi.org/10.1016/j.conb.2008.07.007

Cavanagh, J. F., Bismark, A. J., Frank, M. J., \& Allen, J. J. B. (2011). Larger error signals in major depression are associated with better avoidance learning. Frontiers in Psychology, 2(NOV), 1-6. https:// doi.org/10.3389/fpsyg.2011.00331

Cavanagh, J. F., Cohen, M. X., \& Allen, J. J. B. (2009). Prelude to and resolution of an error: EEG phase synchrony reveals cognitive control dynamics during action monitoring. Journal of Neuroscience, 29(1), 98-105. https://doi.org/10.1523/JNEUROSCI.4137-08.2009

Cavanagh, J. F., Figueroa, C. M., Cohen, M. X., \& Frank, M. J. (2012). Frontal theta reflects uncertainty and unexpectedness during exploration and exploitation. Cerebral Cortex, 22(11), 2575-2586. https://doi.org/10.1093/cercor/bhr332

Cavanagh, J. F., \& Frank, M. J. (2014). Frontal theta as a mechanism for cognitive control. Psychophysiology, 18(8), 414-421. https://doi. org/10.1016/j.tics.2014.04.012

Cavanagh, J. F., Frank, M. J., Klein, T. J., \& Allen, J. J. B. (2010). Frontal theta links prediction errors to behavioral adaptation in reinforcement learning. NeuroImage, 49(4), 3198-3209. https://doi.org/10. 1016/j.neuroimage.2009.11.080

Cavanagh, J. F., Zambrano-Vazquez, L., \& Allen, J. J. B. (2012). Theta lingua franca: A common mid-frontal substrate for action monitoring processes. Psychophysiology, 49(2), 220-238. https://doi.org/ 10.1111/j.1469-8986.2011.01293.x

Coan, J. A., \& Allen, J. J. B. (2004). Frontal EEG asymmetry as a moderator and mediator of emotion. Biological Psychology, 67(1-2), 749. https://doi.org/10.1016/j.biopsycho.2004.03.002

Coan, J. A., Allen, J. J. B., \& McKnight, P. E. (2006). A capability model of individual differences in frontal EEG asymmetry. Biological Psychology, 72(2), 198-207. https://doi.org/10.1016/j.biopsycho. 2005.10.003

Cohen, M. X. (2014). Analyzing neural time series data: Theory and practice. MIT Press, Cambrigde.

Cohen, M. X., \& Donner, T. H. (2013). Midfrontal conflict-related thetaband power reflects neural oscillations that predict behavior. Journal of Neurophysiology, 110(12), 2752-2763. https://doi.org/10.1152/ jn. 00479.2013

Cohen, M. X., Ridderinkhof, K. R., Haupt, S., Elger, C. E., \& Fell, J. (2008). Medial frontal cortex and response conflict: Evidence from human intracranial EEG and medial frontal cortex lesion. Brain Research, 1238, 127-142. https://doi.org/10.1016/j.brainres.2008. 07.114

Cohen, M. X., Wilmes, K., \& van de Vijver, I. (2011). Cortical electrophysiological network dynamics of feedback learning. Trends in 
Cognitive Sciences, 15(12), 558-566. https://doi.org/10.1016/j.tics. 2011.10.004

Collins, A. G. E., Albrecht, M. A., Waltz, J. A., Gold, J. M., \& Frank, M. J. (2017). Interactions among working memory, reinforcement learning, and effort in value-based choice: A new paradigm and selective deficits in schizophrenia. Biological Psychiatry, 82(6), 431-439. https://doi.org/10.1016/j.biopsych.2017.05.017

Davidson, R. J. (1993). Cerebral asymmetry and emotion: Conceptual and methodological conundrums. Cognition and Emotion, 7(1), 115-138. https://doi.org/10.1080/02699939308409180

Davidson, R. J. (1998a). Affective style and affective disorders: Perspectives from affective neuroscience. Cognition and Emotion, 12(3), 307-330. https://doi.org/10.1080/026999398379628

Davidson, R. J. (1998b). Anterior electrophysiological asymmetries, emotion, and depression: Conceptual and methodological conundrums. Psychophysiology, 35(5), S0048577298000134. https://doi. org/10.1017/S0048577298000134

Davidson, R. J. (2004). What does the prefrontal cortex "do" in affect: Perspectives on frontal EEG asymmetry research. Biological Psychology, 67(1-2), 219-233. https://doi.org/10.1016/j. biopsycho.2004.03.008

Davidson, R. J., Ekman, P., Saron, C. D., Senulis, J. A., \& Friesen, W. V. (1990). Approach-withdrawal and cerebral asymmetry: Emotional expression and brain physiology: I. Journal of Personality and Social Psychology, 58(2), 330-341. https://doi.org/10.1037/00223514.58.2.330

Dayan, P., \& Berridge, K. C. (2014). Model-based and model-free Pavlovian reward learning: Revaluation, revision, and revelation. Cognitive, Affective, \& Behavioral Neuroscience, 14(2), 473-492. https://doi.org/10.3758/s13415-014-0277-8

De Raedt, R., Vanderhasselt, M. A., \& Baeken, C. (2015). Neurostimulation as an intervention for treatment resistant depression: From research on mechanisms towards targeted neurocognitive strategies. Clinical Psychology Review https://doi. org/10.1016/j.cpr.2014.10.006

Debener, S., Beauducel, A., Nessler, D., Brocke, B., Heilemann, H., \& Kayser, J. (2000). Is resting anterior EEG alpha asymmetry a trait marker for depression? Neuropsychobiology, 41(1), 31-37. https:// doi.org/10.1159/000026630

Delorme, A., \& Makeig, S. (2004). EEGLAB: an open source toolbox for analysis of single-trial EEG dynamics including independent component analysis. Journal of Neuroscience Methods, 134(1), 9-21. https://doi.org/10.1016/j.jneumeth.2003.10.009

Donner, T. H., \& Siegel, M. (2011). A framework for local cortical oscillation patterns. Trends in Cognitive Sciences https://doi.org/10.1016/ j.tics.2011.03.007

Duprat, R., Desmyter, S., Rudi, D. R., Van Heeringen, K., Van Den Abbeele, D., Tandt, H., ... Baeken, C. (2016). Accelerated intermittent theta burst stimulation treatment in medication-resistant major depression: A fast road to remission? Journal of Affective Disorders, 200(June), 6-14. https://doi.org/10.1016/j.jad.2016.04.015

Endrass, T., \& Ullsperger, M. (2014). Specificity of performance monitoring changes in obsessive-compulsive disorder. Neuroscience and Biobehavioral Reviews, 46(P1), 124-138. https://doi.org/10.1016/j. neubiorev.2014.03.024

Eppinger, B., Kray, J., Mock, B., \& Mecklinger, A. (2008). Better or worse than expected? Aging, learning, and the ERN. Neuropsychologia, 46(2), 521-539. https://doi.org/10.1016/j. neuropsychologia.2007.09.001

Fava, M. (2003). Diagnosis and definition of treatment-resistant depression. Biological Psychiatry https://doi.org/10.1016/S0006-3223(03) 00231-2

Foti, D., Carlson, J. M., Sauder, C. L., \& Proudfit, G. H. (2014). Reward dysfunction in major depression: Multimodal neuroimaging evidence for refining the melancholic phenotype. NeuroImage, 101, 50-58. https://doi.org/10.1016/j.neuroimage.2014.06.058
Foti, D., Weinberg, A., Bernat, E. M., \& Proudfit, G. H. (2015). Anterior cingulate activity to monetary loss and basal ganglia activity to monetary gain uniquely contribute to the feedback negativity. Clinical Neurophysiology, 126(7), 1338-1347. https://doi.org/10. 1016/j.clinph.2014.08.025

Fox, M. D., Buckner, R. L., White, M. P., Greicius, M. D., \& PascualLeone, A. (2012). Efficacy of transcranial magnetic stimulation targets for depression is related to intrinsic functional connectivity with the subgenual cingulate. Biological Psychiatry, 72(7), 595-603. https://doi.org/10.1016/j.biopsych.2012.04.028

Frank, M. J., Woroch, B. S., \& Curran, T. (2005). Error-related negativity predicts reinforcement learning and conflict biases. Neuron, 47(4), 495-501. https://doi.org/10.1016/j.neuron.2005.06.020

Gard, D. E., Gard, M. G., Kring, A. M., \& John, O. P. (2006). Anticipatory and consummatory components of the experience of pleasure: A scale development study. Journal of Research in Personality, 40(6), 1086-1102. https://doi.org/10.1016/j.jrp.2005. 11.001

Gheza, D., De Raedt, R., Baeken, C., \& Pourtois, G. (2018). Integration of reward with cost anticipation during performance monitoring revealed by ERPs and EEG spectral perturbations. NeuroImage, 173. https://doi.org/10.1016/j.neuroimage.2018.02.049

Gotlib, I. H., Ranganath, C., \& Rosenfeld, J. P. (1998). Frontal EEG alpha asymmetry, depression, and cognitive functioning. Cognition \& Emotion, 12(3), 449-478. https://doi.org/10.1080/ 026999398379673

Gray, C. M., \& Singer, W. (1989). Stimulus-specific neuronal oscillations in orientation columns of cat visual cortex. Proceedings of the National Academy of Sciences, 86(5), 1698-1702. https://doi.org/ 10.1073/pnas.86.5.1698

Greden, J. F. (2001). The burden of disease for treatment-resistant depression. The Journal of Clinical Psychiatry, 62 Suppl 1(suppl 16), 26 31. Retrieved from http://www.ncbi.nlm.nih.gov/pubmed/11480881

Hajihosseini, A., \& Holroyd, C. B. (2013). Frontal midline theta and N200 amplitude reflect complementary information about expectancy and outcome evaluation. Psychophysiology, 50(6), 550-562. https://doi.org/10.1111/psyp. 12040

Hamilton, M. (1980). Rating depressive patients. Journal of Clinical Psychiatry, 41(12 II), 21-24.

Harmon-Jones, E., \& Allen, J. J. (1997). Behavioral activation sensitivity and resting frontal EEG asymmetry: covariation of putative indicators related to risk for mood disorders. Journal of Abnormal Psychology, 106(1), 159-163. https://doi.org/10.1037/0021-843X. 106.1.159

Harmon-Jones, E., \& Gable, P. A. (2017). On the role of asymmetric frontal cortical activity in approach and withdrawal motivation: An updated review of the evidence. Psychophysiology, (December 2016). https://doi.org/10.1111/psyp.12879

Hasler, G., Drevets, W. C., Manji, H. K., \& Charney, D. S. (2004). Discovering endophenotypes for major depression. Neuropsychopharmacology https://doi.org/10.1038/sj.npp.1300506

Henriques, J. B., \& Davidson, R. J. (1991). Left frontal hypoactivation in depression. Journal of Abnormal Psychology, 100(4), 535-545. https://doi.org/10.1037/0021-843X.100.4.535

Henriques, J. B., \& Davidson, R. J. (2000). Decreased responsiveness to reward in depression. Cognition \& Emotion, 14(5), 711-724. https:// doi.org/10.1080/02699930050117684

Holroyd, C. B., \& Coles, M. G. H. (2002). The neural basis of human error processing: Reinforcement learning, dopamine, and the errorrelated negativity. Psychological Review, 109(4), 679-709. https:// doi.org/10.1037/0033-295X.109.4.679

Holroyd, C. B., Pakzad-Vaezi, K. L., \& Krigolson, O. E. (2008). The feedback correct-related positivity: Sensitivity of the event-related brain potential to unexpected positive feedback. Psychophysiology, 45(5), 688-697. https://doi.org/10.1111/j.1469-8986.2008.00668.x 
Holroyd, C. B., \& Umemoto, A. (2016). The research domain criteria framework: The case for anterior cingulate cortex. Neuroscience \& Biobehavioral Reviews, 71, 418-443. https://doi.org/10.1016/j. neubiorev.2016.09.021

Jepma, M., \& Nieuwenhuis, S. (2011). Pupil diameter predicts changes in the exploration-exploitation trade-off: Evidence for the adaptive gain theory. Journal of Cognitive Neuroscience, 23(7), 15871596. https://doi.org/10.1162/jocn.2010.21548

Kayser, J., \& Tenke, C. E. (2006). Principal components analysis of Laplacian waveforms as a generic method for identifying ERP generator patterns: I. Evaluation with auditory oddball tasks. Clinical Neurophysiology, 117(2), 348-368.

Kessler, R. C., \& Bromet, E. J. (2013). The epidemiology of depression across cultures. Annual Review of Public Health, 34(1), 119-138. https://doi.org/10.1146/annurev-publhealth-031912-114409

Koban, L., \& Pourtois, G. (2014). Brain systems underlying the affective and social monitoring of actions: An integrative review. Neuroscience and Biobehavioral Reviews, 46(P1), 71-84. https:// doi.org/10.1016/j.neubiorev.2014.02.014

Kunisato, Y., Okamoto, Y., Ueda, K., Onoda, K., Okada, G., Yoshimura, S., ... Yamawaki, S. (2012). Effects of depression on reward-based decision making and variability of action in probabilistic learning. Journal of Behavior Therapy and Experimental Psychiatry, 43(4), 1088-1094. https://doi.org/10.1016/j.jbtep.2012.05.007

Miller, G. A., Crocker, L. D., Spielberg, J. M., Infantolino, Z. P., \& Heller, W. (2013). Issues in localization of brain function: The case of lateralized frontal cortex in cognition, emotion, and psychopathology. Frontiers in Integrative Neuroscience, 7(January), 1-9. https:// doi.org/10.3389/fnint.2013.00002

Moscovitch, D. A., Santesso, D. L., Miskovic, V., McCabe, R. E., Antony, M. M., \& Schmidt, L. A. (2011). Frontal EEG asymmetry and symptom response to cognitive behavioral therapy in patients with social anxiety disorder. Biological Psychology, 87(3), 379-385. https://doi.org/10.1016/j.biopsycho.2011.04.009

Nalborczyk, L., Batailler, C., Loevenbruck, H., Vilain, A., \& Bürkner, P. C. (in press). An Introduction to Bayesian Multilevel Models Using brms: A Case Study of Gender Effects on Vowel Variability in Standard Indonesian. Journal of Speech, Language, and Hearing Research, (in press).

Nelson, B. D., Kessel, E. M., Klein, D. N., \& Shankman, S. A. (2017). Depression symptom dimensions and asymmetrical frontal cortical activity while anticipating reward. Psychophysiology, (April 2017), 1-14. https://doi.org/10.1111/psyp.12892

Nigbur, R., Cohen, M. X., Ridderinkhof, K. R., \& Stürmer, B. (2012). Theta dynamics reveal domain-specific control over stimulus and response conflict. Journal of Cognitive Neuroscience, 24(5), 1264 1274. https://doi.org/10.1162/jocn_a_00128

Nusslock, R., Walden, K., \& Harmon-Jones, E. (2015). Asymmetrical frontal cortical activity associated with differential risk for mood and anxiety disorder symptoms: An RDoC perspective. International Journal of Psychophysiology, 98(2), 249-261. https://doi.org/10.1016/j.ijpsycho.2015.06.004

Olvet, D. M., \& Hajcak, G. (2009). The error-related negativity (ERN) and psychopathology: toward an endophenotype. Clinical Psychology Review, 28(8), 1343-1354. https://doi.org/10.1016/j. cpr.2008.07.003.The

Perrin, F., Pernier, J., Bertrand, O., \& Echallier, J. F. (1989). Spherical splines for scalp potential and current density mapping. Electroencephalography and Clinical Neurophysiology, 72(2), 184-187. https://doi.org/10.1016/0013-4694(89)90180-6

Pizzagalli, D. A. (2014). Depression, Stress, and Anhedonia: Toward a Synthesis and Integrated Model. Annual Review of Clinical Psychology, 10(1), 393-423. https://doi.org/10.1146/annurevclinpsy-050212-185606

Pizzagalli, D. A., Iosifescu, D., Hallett, L. A., Ratner, K. G., \& Fava, M. (2008). Reduced hedonic capacity in major depressive disorder:
Evidence from a probabilistic reward task. Journal of Psychiatric Research, 43(1), 76-87. https://doi.org/10.1016/j.jpsychires.2008. 03.001

Pizzagalli, D. A., Sherwood, R. J., Henriques, J. B., \& Davidson, R. J. (2005). Frontal brain asymmetry and reward responsiveness: A source-localization study. Psychological Science, 16(10), 805-813. https://doi.org/10.1111/j.1467-9280.2005.01618.x

Proudfit, G. H. (2015). The reward positivity: From basic research on reward to a biomarker for depression. Psychophysiology, 52(4), 449-459. https://doi.org/10.1111/psyp.12370

R Core Team. (2017). R. R Core Team. https://doi.org/3-900051-14-3

Rossion, B., \& Pourtois, G. (2004). Revisiting Snodgrass and Vanderwart's object pictorial set: The role of surface detail in basic-level object recognition. Perception, 33(2), 217-236. https:// doi.org/10.1068/p5117

Rush, A. J., Thase, M. E., \& Dubé, S. (2003). Research issues in the study of difficult-to-treat depression. Biological Psychiatry https://doi.org/ 10.1016/S0006-3223(03)00088-X

Rutledge, R. B., Moutoussis, M., Smittenaar, P., Zeidman, P., Taylor, T., Hrynkiewicz, L., ... Dolan, R. J. (2017). Association of neural and emotional impacts of reward prediction errors with major depression. JAMA Psychiatry, 74(8), 790. https://doi.org/10.1001/ jamapsychiatry.2017.1713

Salamone, J. D., \& Correa, M. (2012). The mysterious motivational functions of mesolimbic dopamine. Neuron, 76(3), 470-485. https://doi. org/10.1016/j.neuron.2012.10.021

Salamone, J. D., Correa, M., Farrar, A., \& Mingote, S. M. (2007). Effortrelated functions of nucleus accumbens dopamine and associated forebrain circuits. Psychopharmacology, 191(3), 461-482. https:// doi.org/10.1007/s00213-006-0668-9

Salamone, J. D., Correa, M., Nunes, E. J., Randall, P. A., \& Pardo, M. (2012). The behavioral pharmacology of effort-related choice behavior: Dopamine, adenosine and beyond. Journal of the Experimental Analysis of Behavior, 97(1), 125-46. https://doi.org/ 10.1901/jeab.2012.97-125

Shankman, S. A., Klein, D. N., Tenke, C. E., \& Bruder, G. E. (2007). Reward sensitivity in depression: A biobehavioral study. Journal of Abnormal Psychology, 116(1), 95-104. https://doi.org/10.1037/ 0021-843X.116.1.95

Sheehan, D. V, Lecrubier, Y., Sheehan, K. H., Amorim, P., Janavs, J., Weiller, E., ... Dunbar, G. C. (1998). The Mini-International Neuropsychiatric Interview (M.I.N.I.): The development and validation of a structured diagnostic psychiatric interview for DSM-IV and ICD-10. The Journal of Clinical Psychiatry, 59(Suppl 2), 2257.

Smith, E. E., Cavanagh, J. F., \& Allen, J. J. B. (2017). Intracranial source activity (eLORETA) related to scalp-level asymmetry scores and depression status. Psychophysiology, (July 2017), 1-16. https://doi. org/10.1111/psyp.13019

Smith, E. E., Reznik, S. J., Stewart, J. L., \& Allen, J. J. B. (2017). Assessing and conceptualizing frontal EEG asymmetry: An updated primer on recording, processing, analyzing, and interpreting frontal alpha asymmetry. International Journal of Psychophysiology, 111, 98-114. https://doi.org/10.1016/j.ijpsycho.2016.11.005

Smith, E. H., Banks, G. P., Mikell, C. B., Cash, S. S., Patel, S. R., Eskandar, E. N., \& Sheth, S. A. (2015). Frequency-dependent representation of reinforcement-related information in the human medial and lateral prefrontal cortex. Journal of Neuroscience, 35(48), 15827-15836. https://doi.org/10.1523/JNEUROSCI.1864-15.2015

Snaith, R. P., Hamilton, M., Morley, S., Humayan, A., Hargreaves, D., \& Trigwell, P. (1995). A scale for the assessment of hedonic tone. The Snaith-Hamilton Pleasure Scale. British Journal of Psychiatry, 167(JULY), 99-103. https://doi.org/10.1192/bjp.167.1.99

Stewart, J. L., Bismark, A. W., Towers, D. N., Coan, J. A., \& Allen, J. J. B. (2010). Resting frontal EEG asymmetry as an endophenotype for depression risk: Sex-specific patterns of frontal brain asymmetry. 
Journal of Abnormal Psychology, 119(3), 502-512. https://doi.org/ 10.1037/a0019196

Stewart, J. L., Coan, J. A., Towers, D. N., \& Allen, J. J. B. (2011). Frontal EEG asymmetry during emotional challenge differentiates individuals with and without lifetime major depressive disorder. Journal of Affective Disorders, 129(1-3), 167-174. https://doi.org/10.1016/j. jad.2010.08.029

Stewart, J. L., Coan, J. A., Towers, D. N., \& Allen, J. J. B. (2014). Resting and task-elicited prefrontal EEG alpha asymmetry in depression: Support for the capability model. Psychophysiology, 51(5), 446455. https://doi.org/10.1111/psyp.12191

Sutton, R. S., \& Barto, A. G. (2018). Reinforcement learning: An introduction. MIT Press: Cambridge, MA.

Tallon-Baudry, \& Bertrand. (1999). Oscillatory gamma activity in humans and its role in object representation. Trends in Cognitive Sciences, 3(4), 151-162. https://doi.org/10.1016/S1364-6613(99) 01299-1

The MathWorks Inc. (2013). MATLAB. Natick, MA, USA.

Thibodeau, R., Jorgensen, R. S., \& Kim, S. (2006). Depression, anxiety, and resting frontal EEG asymmetry: A meta-analytic review. Journal of Abnormal Psychology, 115(4), 715-729. https://doi.org/ 10.1037/0021-843X.115.4.715

Thomsen, K. R. (2015). Measuring anhedonia: Impaired ability to pursue, experience, and learn about reward. Frontiers in Psychology, 6(September), 1409. https://doi.org/10.3389/fpsyg.2015.01409

Treadway, M. T., Bossaller, N., Shelton, R. C., \& Zald, D. H. (2012). Effort-based decision-making in major depressive disorder: A translational model of motivational anhedonia. Journal of Abnormal Psychology, 121(3), 553-558. https://doi.org/10.1037/a0028813

Treadway, M. T., \& Zald, D. H. (2011). Reconsidering anhedonia in depression: Lessons from translational neuroscience. Neuroscience and Biobehavioral Reviews, 35(3), 537-555. https://doi.org/10. 1016/j.neubiorev.2010.06.006

Ullsperger, M., Fischer, A. G., Nigbur, R., \& Endrass, T. (2014). Neural mechanisms and temporal dynamics of performance monitoring. Trends in Cognitive Sciences, 18(5), 259-267. https://doi.org/10. 1016/j.tics.2014.02.009

Vaidyanathan, U., Nelson, L. D., \& Patrick, C. J. (2012). Clarifying domains of internalizing psychopathology using neurophysiology. Psychological Medicine, 42(03), 447-459. https://doi.org/10.1017/ S0033291711001528 van de Vijver, I., Cohen, M. X., \& Ridderinkhof, K. R. (2014). Aging affects medial but not anterior frontal learning-related theta oscillations. Neurobiology of Aging, 35(3), 692-704. https://doi.org/10. 1016/j.neurobiolaging.2013.09.006

van de Vijver, I., Ridderinkhof, K. R., \& Cohen, M. X. (2011). Frontal oscillatory dynamics predict feedback learning and action adjustment. Journal of Cognitive Neuroscience, 23(12), 4106-4121. https://doi.org/10.1162/jocn_a_00110

van der Vinne, N., Vollebregt, M. A., van Putten, M. J. A. M., \& Arns, M. (2017). Frontal alpha asymmetry as a diagnostic marker in depression: Fact or fiction? A meta-analysis. NeuroImage: Clinical, 16(July), 79-87. https://doi.org/10.1016/j.nicl.2017.07.006

Vrieze, E., Pizzagalli, D. A., Demyttenaere, K., Hompes, T., Sienaert, P., De Boer, P., ... Claes, S. (2013). Reduced reward learning predicts outcome in major depressive disorder. Biological Psychiatry, 73(7), 639-645. https://doi.org/10.1016/j.biopsych.2012.10.014

Wang, C. (2005). Responses of human anterior cingulate cortex microdomains to error detection, conflict monitoring, stimulus-response mapping, familiarity, and orienting. Journal of Neuroscience, 25(3), 604-613. https://doi.org/10.1523/JNEUROSCI.4151-04.2005

Weinberg, A., Liu, H., Hajcak, G., \& Shankman, S. A. (2015). Blunted neural response to rewards as a vulnerability factor for depression: Results from a family study. Journal of Abnormal Psychology, 124(4), 878-889. https://doi.org/10.1037/abn0000081

Weinberg, A., Riesel, A., \& Hajcak, G. (2012). Integrating multiple perspectives on error-related brain activity: The ERN as a neural indicator of trait defensive reactivity. Motivation and Emotion, 36(1), 84-100. https://doi.org/10.1007/s11031-0119269-y

Weinberg, A., \& Shankman, S. A. (2016). Blunted reward processing in remitted melancholic depression. Clinical Psychological Science, 2167702616633158. https://doi.org/10.1177/2167702616633158

Whitton, A. E., Kakani, P., Foti, D., Van’t Veer, A., Haile, A., Crowley, D. J., \& Pizzagalli, D. A. (2016). Blunted neural responses to reward in remitted major depression: A high-density event-related potential study. Biological Psychiatry: Cognitive Neuroscience and Neuroimaging, 1(1), 87-95. https://doi.org/10.1016/j.bpsc.2015. 09.007

Yeung, N., Holroyd, C. B., \& Cohen, J. D. (2005). ERP correlates of feedback and reward processing in the presence and absence of response choice. Cerebral Cortex, 15(5), 535-544. https://doi.org/ 10.1093/cercor/bhh153 